\title{
Drawing mechanisms for top coal in longwall top coal caving (LTCC): A review of two decades of literature
}

\author{
Jiachen Wang ${ }^{1,3} \cdot$ Shengli Yang ${ }^{1,3} \cdot$ Weijie Wei ${ }^{1,3} \cdot$ Jinwang Zhang ${ }^{1,3} \cdot$ \\ Zhengyang Song ${ }^{2,3}$ (B)
}

Received: 25 March 2021/Revised: 7 June 2021 / Accepted: 29 June 2021 / Published online: 21 July 2021

(C) The Author(s) 2021

\begin{abstract}
This review details the state of the art in research on top coal drawing mechanisms in Longwall top coal caving (LTCC) by examining the relevant literature over the last two decades. It starts with an introduction of the brief history and basic procedures of LTCC. The framework of research on the drawing mechanism, basic concepts, and some theoretical models of LTCC are detailed in sect. research framework of top coal drawing mechanism. The authors note that the Top coal drawbody (TCD), Top coal boundary (TCB) and Top coal recovery ratio (TCRR) are key factors in the drawing mechanism. The Body-boundary-ratio (BBR) research system has been the classic framework for research over the last 20 years. The modified Bergmark-Roos model, which considers the effects of the supporting rear canopy, flowing velocity of top coal, and its shape factor, is optimal for characterizing the TCD. A 3D model to describe the TCB that considers the thicknesses of the coal seam and roof strata is reviewed. In sect. physical testing and numerical simulation, the physical tests and numerical simulations in the literature are classified for ease of bibliographical review, and classic conclusions regarding the drawing mechanism of top coal are presented and discussed with elaborate illustrations and descriptions. The deflection of the TCD is noted, and is caused by the shape of the rear canopy. The inclined coal seam always induces a larger TCD, and a deflection in the TCD has also been observed in it. The effects of the drawing sequence and drawing interval on the TCRR are reviewed, where a long drawing interval is found to lead to significant loss of top coal. Its flowing behavior and velocity distribution are also presented. Sect. practical applications of drawing mechanisms for LTCC mines 4 summarizes over 10 cases where the TCRR of LTCC mines improved due to the guidance of the drawing mechanism. The final section provides a summary of the work here and some open questions. Prospective investigations are highlighted to give researchers guidance on promising issues in future research on LTCC.
\end{abstract}

Keywords Longwall top coal caving (LTCC) · Top coal drawing · Top coal drawbody (TCD) · Top coal boundary (TCB) · Top coal recovery ratio (TCRR)

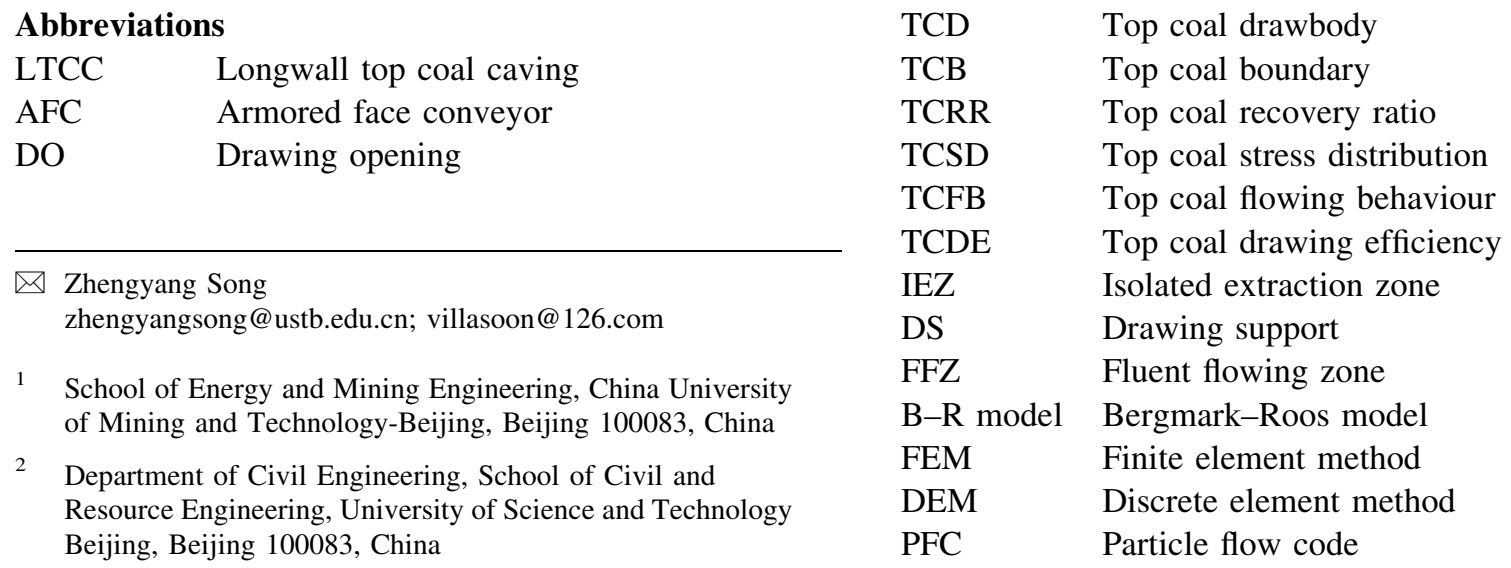




\section{Introduction}

Longwall top coal caving (LTCC) is a special type of mining method commonly used to extract coal seams thicker than $4.5 \mathrm{~m}$ (Wang et al. 2018a). This method originated in France and Eastern Europe in the 1950s for extracting coal pillars from marginal areas of coalfields (Wang et al. 2014). LTCC is mainly used in China these days, and has been applied in a few cases in Australia, Vietnam, Russia, Turkey, and Slovenia (Le et al. 2017, 2019; Song et al. 2020; Jangara and Ozturk 2021). According to the China National Bureau of Statistics, the country produced 3.75 billion tons of raw coal in 2019, and mined the largest amount of it using LTCC in the world. With 30 years of development, LTCC has become a representative mining technology in China (Wang 2014, 2018). Figure 1a illustrates the basic operations of LTCC. The coal seam in LTCC consists of two parts: 1) bottom coal seam, and 2) top coal. The bottom part is undercut by a shearer located on an armored face conveyor. This is a typical layout in conventional longwall mining. Top coal is fractured by the effect of mining-induced stress (Alehossein and Poulsen 2010; Sajjad et al. 2019; Hao et al. 2020), repeated movement of the shield beams (Xie and Zhao 2009a; Wang et al. 2015c), or is artificially broken through hydraulic fracturing (Huang et al. 2015, 2018) or pre-blasting (Lai et al. 2014). The Drawing opening (DO) is embedded into the rear of the hydraulic support in LTCC. Once the DO is opened, the fractured top coal flows to the Armored face conveyor (AFC) through the DO. This process is often called top coal drawing. From the viewpoint of mechanics, the top coal drawing process is dominated by gravity and frictional forces. This indicates that research on the top coal drawing mechanism should be conducted within the scope of gravitational flow. In metal mines, block caving is often used. It is an underground mining method that uses gravity to exploit massive, steeply dipping ore bodies located at a considerable depth. Many studies on top coal drawing mechanisms often use gravitational flow in block caving for reference (Wang et al. 2015d; Jin et al. 2016, 2017; Song et al. 2018; Song and Konietzky 2019). This study reviews and summarizes of top coal drawing mechanisms in LTCC excluding those based on gravitational flow in block caving. The reader interested in research on gravitational flow can refer to Melo et al. (2007, 2008, 2009), Castro et al. (2016), and Sánchez et al. (2019). The primary objective of this work is to present a comprehensive account of the state of the art in research on top coal drawing mechanisms, and herein lies its originality. The authors use the literature over the last 20 years to provide a global perspective of published articles and highlight potential areas for future research on LTCC.

The remainder of this paper is organized as follows: Sect. 2 summarizes the framework of research on top coal drawing mechanisms. Some theoretical models, basic concepts, and theories are summarized and discussed. Section 3 provides a comprehensive summary of the relevant physical testing and numerical simulations. The corresponding experimental and numerical results are discussed in detail rather than merely listed. Section 4 presents more than 10 practical cases of the application of the drawing mechanism. Section 5 contains a discussion of outstanding questions and gray areas as an incentive for future work on LTCC. The current limitations and prevalent perspectives are also noted.

\section{Research framework of top coal drawing mechanism}

\subsection{Basic conceptions}

\subsubsection{Top coal drawbody (TCD)}

As shown in Fig. 1, the Top coal drawbody (TCD) refers to the drawn out top coal blocks before the DO closes
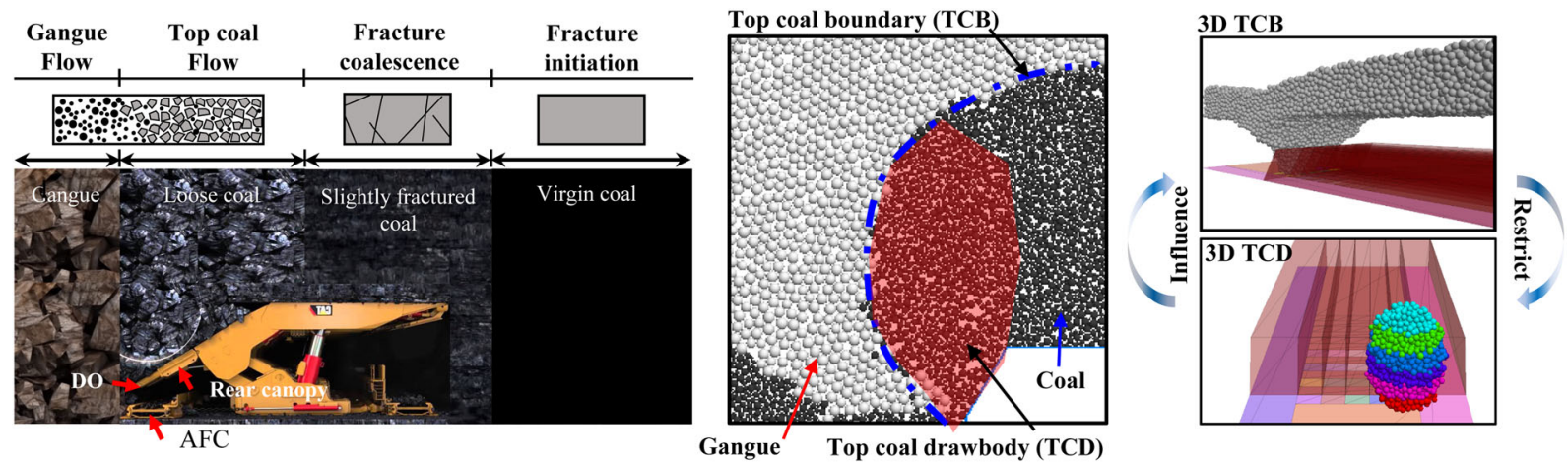

Fig. 1 Illustrations of LTCC operation, top coal boundary (TCB), and top coal drawbody (TCD) 
(termination of the drawing procedure)—see the red zones in Fig. 1. According to the practical principle applied to LTCC mines, the DO should be immediately closed when the gangue (waste rocks above the top coal) flows out. For current, the close of DO is mainly dependent on the experiences of miner, however, the intelligent control of DO has been applied in a few cases in China. The cameras set at the end of the rear canopy will automatically recognize the gangue by using the picture analysis algorithm, so the close of the drawing opening can be realized automatically, this technology has now applied in some LTCC mines and will be soon used by more LTCC mines in near future. Dominated by gravity, the shape of the TCD quite is similar to the isolated extraction zone (IEZ) in block caving. However, the hydraulic drawing support (DS) used in LTCC leads to the formation of a distinct boundary in comparison with the block caving where no DS is used, and the rear canopy of the DS influences the shape of the TCB (Wang and Zhang 2015; Wang et al. 2016d). Furthermore, the DS moves forward after each drawing procedure and the TCB evolves with the advance of the DS. In summary, top coal drawing is a continuous and interactive process (Song et al. 2020).

\subsubsection{Top coal boundary (TCB)}

The top coal boundary (TCB) is the continuous interface between the top coal and the gangue (Wei et al. 2018). Because top coal is drawn under zones of the TCB, the process is determined and controlled by the shape of the TCB. The blue line in Fig. 1 indicates the outline of the TCB in a $2 \mathrm{D}$ version. A $3 \mathrm{D}$ TCB and TCD are plotted in the right part of Fig. 1. It also shows the interrelations between the TCB and the TCD. The TCB directly impacts and restricts the volume of the TCD, which impacts the shape of TCB in the next drawing procedure in turn.

\subsubsection{Top coal recovery ratio (TCRR)}

The top coal recovery ratio (TCRR) refers to the percentage of recovered (drawn out) top coal divided by the total top coal in designated zones. The TCRR is an effective index to characterize the loss of top coal. A higher TCRR can yield significant economy benefit and little resource loss.

\subsubsection{Top coal stress distribution (TCSD)}

The stress distribution of top coal refers to the complex contact forces between the fractured blocks of top coal. Interlocking between the blocks always occurs when the localized contact force is large. Arching then occurs, and drawing is terminated due to the effects of jamming and arching.

\subsubsection{Top coal flowing behavior (TCFB) and drawing efficiency $(T C D E)$}

Top coal flowing behavior (TCFB) represents features of the flow of the top coal and gangue. It includes the flow trajectory and velocity of top coal. Top coal drawing efficiency (TCDE) is an index to evaluate the extent of fluency of the top coal blocks. The more fluent is the drawing, the higher is the efficiency in LTCC.

\subsection{Key issues in top coal drawing mechanism}

Based on the relevant literature over the last two decades (2000-2020), Fig. 2 shows the key issues, questions, and expected goals in research on top coal drawing in LTCC. The research framework that has largely been used is also shown in Fig. 2.

The contributions made by different researchers are summarized in Table 1 based on literature in the last two decades (2000-2020). It presents studies according to issue. The main contributions or conclusions are listed in the rightmost column.

\subsection{Theoretical models}

This section reviews the theoretical models proposed to investigate the top coal drawing mechanism, and these include models of the TCD and TCB.

\subsubsection{Models of TCD}

2.3.1.1 Bergmark-Roos $(B-R)$ model The BergmarkRoos (B-R) model is commonly used to describe gravitational flow (Rustan 2000). This model was first used in block caving mining to theoretically characterize the shape of the IEZ (Melo et al. 2007; Tao et al. 2010; Song et al. 2018). Two basic assumptions in the B-R model are as follows: 1) The blocks move in straight lines from their resting point to the DO. 2) The blocks are dominated by gravitational and frictional forces (Kuchta 2002). Figure 3a shows the coordinate system of the original B-R model, and its equations are as follows (Melo et al. 2007; Wang et al. 2016b):

$$
\begin{aligned}
& r_{\max }=r_{D}+\left(\frac{g t^{2}}{2}\right)\left(1-\cos \theta_{G}\right) \\
& r_{0}\left(\theta, r_{\max }\right)=\left(r_{\max }-r_{D}\right) \frac{\left(\cos \theta-\cos \theta_{G}\right)}{1-\cos \theta_{G}}
\end{aligned}
$$

Figure $3 \mathrm{a}$, in which $\theta_{G}$ is the maximum allowed angle of motion, indicates that the particle can move only when 

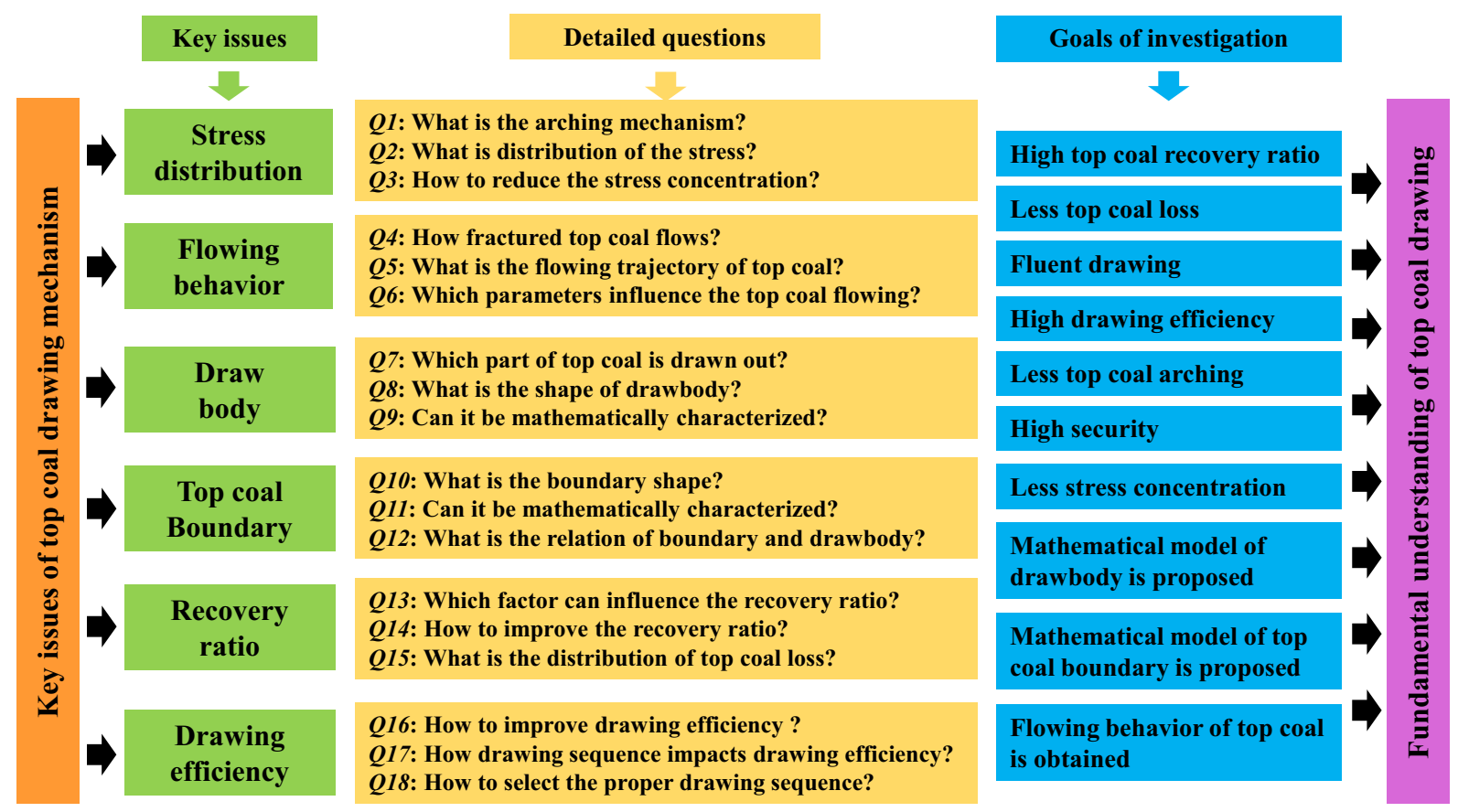

Fig. 2 Research framework of the top coal drawing mechanism

angle is smaller than $\theta_{G} . r_{\max }$ is the distance between the farthest resting point and the DO, $t$ is drawing time, and $\theta$ is the angle of motion. $r_{D}$ is the distance between the origin of the polar coordinates and the aperture. According to Eq. (1), the resting points with different moving angles can be calculated, following which the boundary of the TCD can be obtained by linking discrete boundary points. However, the original B-R model does not consider the effect of the rear canopy of the DS on the TCB. The modified B-R is shown in Eq. (2), obtained after considering the effect of the shape of the rear canopy (Wang et al. 2016b, d; Zhang et al. 2019). As shown in Fig. 3b, the maximum allowed angle close to the rear canopy (near side of rear canopy) is set to $90^{\circ}-\beta$ ( $\beta$ is the angle of the rear canopy with respect to the horizontal direction), and is different from the original B-R model, while the far side of rear canopy still uses $\theta_{G}$ as the maximum allowed angle:

$$
\begin{aligned}
r(\theta)= & r\left(r_{\max }-r_{D}\right) \frac{(\cos \theta-\cos \theta)}{1-\cos \theta_{G}} \\
& +r_{D}(\text { far side of rear canopy }) \\
r(\theta)= & \left(r_{\max }-r_{D}\right) \frac{(\cos \theta-\cos (90-\beta))}{1-\cos (90-\beta)} \\
& +r_{D}(\text { near side of rear canopy })
\end{aligned}
$$

Even though it considers the effect of the rear canopy, Eq. (2) still has two major disadvantages (Song et al. 2020)
(1) The TCB exhibits a discontinuity at the point connecting the far side and the near side.

(2) The model significantly underestimates the drawing time.

The first shortcoming occurs because of non-uniform maximum allowable angles in the far and near sides. The underestimation of the drawing time is induced by ignoring the interlocking between the top coal blocks. To overcome these flaws, the model is further amended by introducing a velocity correction factor, $k$, see Eq. (3). In Eq. (3), $k_{F}$ and $k_{N}$ are, respectively, the velocity correction factors for the far and near sides of the rear canopy, $t_{F}$ and $t_{N}$ are the theoretical flowing times at the far and near side, respectively, and $t$ is the actual flowing time of the top coal blocks that can be measured through the physical tests:

$$
\begin{aligned}
r_{\max } & =r_{D}+k_{F} \text { or }\left(k_{N}\right) \frac{g t^{2}}{2}\left(1-\cos \theta_{G}\right) ; k_{F}=t_{F}^{2} / t^{2}, k_{N}=t_{N}^{2} / t^{2} \\
r(\theta) & =\left(r_{\max }-r_{D}\right) \frac{\left(\cos \theta-\cos \theta_{G}\right)}{1-\cos \theta_{G}}+r_{D}(\text { far side of rear canopy }) \\
r(\theta) & =\left(r_{\max }-r_{D}\right) \frac{(\cos \theta-\cos (90-\beta))}{1-\cos (90-\beta)}+r_{D}(\text { near side of rear canopy })
\end{aligned}
$$

Equation (3) can address the discontinuities of the TCD and amend the unrealistic drawing time encountered in Eq. (2). However, it still has one significant defect: Once the thickness of a coal seam is fixed, the shape of the TCD is also fixed without considering other factors, such as the shape and diameters of the top coal blocks. The model 
Table 1 Summary of research on the drawing mechanism in LTCC

\begin{tabular}{|c|c|c|}
\hline Item & Main contribution & Reference \\
\hline TCD & The deflection phenomenon of drawbody was observed & Wang et al. (2015c) \\
\hline TCD & Theoretical model of drawbody was proposed & Wang et al. (2016b) \\
\hline TCD & Theoretical model of drawbody was derived based on stochastic medium theory & Zhu et al. (2018) \\
\hline TCD & Theoretical model of drawbody in steep seam was derived & Zhang et al. (2018a) \\
\hline TCD & Effect of top coal block size on drawbody was investigated & Wang et al. (2020a) \\
\hline TCD & Effect of upward angle on the drawbody was studied & Yang et al. (2016) \\
\hline TCD & A research system for drawing mechanism was proposed & Wang et al. (2016d) \\
\hline TCD & Drawbody shape after support advance was considered & Song and Konietzky (2019) \\
\hline TCD & Drawbody exposed to sectional fractured top coal was studied & Song et al. (2020) \\
\hline TCD & Effect of size distribution of granular top coal on drawbody was studied & Wang et al. (2019a) \\
\hline TCD & Drawbody was in the form of arc-shaped strips in front of working face & Zhang et al. (2016) \\
\hline TCD & Effects of caving-mining ratio on drawbody was studied & Zhang et al. (2015b) \\
\hline TCD & Drawbody of top-coal showed an irregularly deflected ellipsoid & Zhang et al. (2019) \\
\hline TCD & Drawbody in LTCC was a cut-variant ellipsoid & Wang and Zhang (2015) \\
\hline TCD & Drawbody in pre-broken condition was studied & Klishin1 et al. (2018) \\
\hline TCD & Theoretical model of drawbody in inclined seam was proposed & Wang et al. (2019b) \\
\hline TCD & Model of drawbody was proposed for ultra-thick coal seam & Wang et al. (2015b) \\
\hline TCD & Effect of top coal shape on drawbody was studied & Song et al. (2018) \\
\hline TCD & Drawbody shape was numerically revealed & Wang et al. (2013) \\
\hline TCB & Theoretical model of TCB was derived & Wei et al. (2018) \\
\hline TCB & TCB equation in inclined seam was derived & Zhang et al. (2018a) \\
\hline TCB & The relation between drawbody and TCB was clarified & Wang and Zhang (2015) \\
\hline TCB & TCB in sectional fractured LTCC was revealed & Song et al. (2020) \\
\hline TCB & TCB in steeply inclined condition was derived & Zhang et al. (2014) \\
\hline TCB & The evolution of TCB after support advance was revealed & Song and Konietzky (2019) \\
\hline TCRR & A TCRR prediction model at lab scale was investigated & Wang et al. (2020b) \\
\hline TCRR & A method for improving TCRR was proposed & Wang et al. (2016d) \\
\hline TCRR & TCRR first decreased and then increased with increasing upward angle & Yang et al. (2016) \\
\hline TCRR & LTCC with vibration technology increased the TCRR & Xie and Zhao (2009a) \\
\hline TCRR & Top coal size was a parameter that impacted top coal recovery ratio & Bai et al. (2018) \\
\hline TCRR & The manner of evolution of TCRR with respect to drawing height was revealed & Wang and Zhang (2015) \\
\hline TCSD & A field investigation-based system for top-coal cavability assessment was proposed & Wang et al. (2018b) \\
\hline TCSD & The mechanism of force chain evolution in fractured top coal was revealed & Wang et al. (2020c) \\
\hline TCSD & Stress distribution in LTCC was similar to that in conventional longwall method & Le et al. (2018) \\
\hline TCSD & A new cavability assessment criterion for top coal drawing was proposed & Vakili and Hebblewhite (2010) \\
\hline TCSD & Top coal arching mechanism was revealed from a numerical perspective & Bai et al. (2014) \\
\hline TCSD & Three types of top coal arch structures were formed & Zhang and Liu (2016) \\
\hline TCSD & A comprehensive stress analysis of LTCC was conducted & Alehossein and Poulsen (2010) \\
\hline TCSD & The deformation of top coal in shallow coal seam was studied & Li et al. (2017) \\
\hline TCSD & The in-situ pressure in LTCC was monitored & Si et al. (2015) \\
\hline TCSD & The arching phenomenon in ultra-thick coal seam was studied & Zhang et al. (2018c) \\
\hline TCFB & Top coal flow in the initial drawing process was revealed & Huo et al. (2020) \\
\hline TCFB & Top coal flow in an extremely steep and thick seam was investigated & Miao et al. (2011) \\
\hline TCFB & Effect of parameters on coal-gangue field movements was analyzed & Liu et al. (2009) \\
\hline TCFB & Effect of caving-mining ratio on coal-gangue flow was analyzed & Zhang et al. (2015b) \\
\hline TCDE & To increase operational efficiency, top coal was uniformly fractured & Yasitli and Unver (2005) \\
\hline TCDE & LTCC with vibration technology increased efficiency & Xie and Zhao (2009a) \\
\hline
\end{tabular}


Table 1 continued

\begin{tabular}{lll}
\hline Item & Main contribution & Reference \\
\hline TCDE & Determination of the most effective longwall equipment was discussed & Simsir and Ozfirat (2008) \\
\hline
\end{tabular}

The abbreviations in Table 1 are provided in the Nomenclature

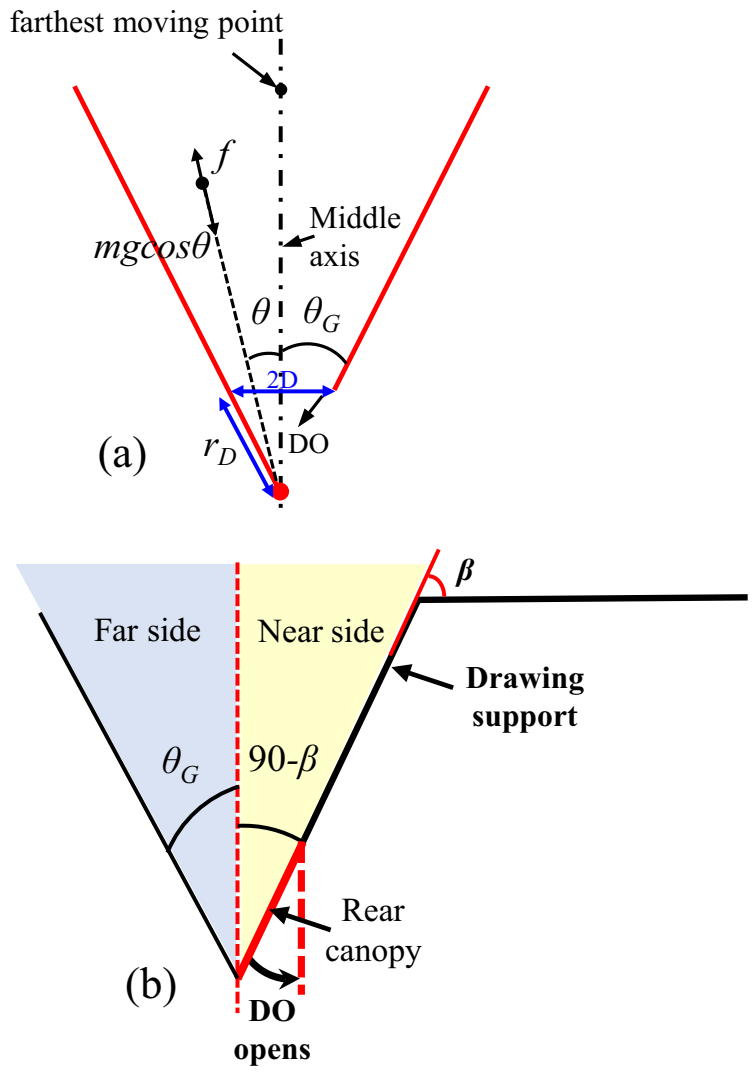

Fig. 3 Polar coordinate system in the original B-R model. A modified B-R model that considers the rear canopy of the DS in LTCC; after Song et al. (2020)

further modified by considering the frictional and shaperelated factors is shown in Eq. (4), where $b$ is a function of the friction and shape of top coal. The values of $b$ at the far side $\left(b_{F}\right)$ and near side $\left(b_{N}\right)$ are different. The modified model in Eq. 4 can adequately characterize the shape of the TCD in numerical simulations (Song et al. 2020).

$$
\begin{aligned}
& r(\theta)=\left(r_{\max }-r_{D}\right) \frac{\left(\cos \theta-\cos \theta_{G}+b_{F}\right)}{1-\cos \theta_{G}+b_{F}} r_{D} ; \\
& b_{F}=G(f, \varphi) ; \theta=90^{\circ}(\text { far side of rear canopy }) r(\theta) \\
& =\left(r_{\max }-r_{D}\right) \frac{\left(\cos \theta-\cos (90-\beta)+b_{N}\right)}{1-\cos (90-\beta)+b_{N}} r_{D} ; \\
& b_{N}=G(f, \varphi)(\text { near side of rear canopy) }
\end{aligned}
$$

2.3.1.2 Kinematic model The kinematic model is a classic means of characterizing the flow of granular particles, and was proposed by Nedderman and Tüzün (1979). The basic assumptions for the kinematic model are as follows:

(1) The bottom of the DO is flat and the particles are loosely packed. In the 3D kinematic model, the particle velocity is shown as in Eq. (5):

$$
V_{x}=-D_{p} \frac{\partial V_{z}}{\partial x}, V_{y}=-D_{p} \frac{\partial V_{z}}{\partial y}
$$

(2) The continuous flow of granular particles is regarded as a fluid state, and thus the movements of particles meet the continuity equation in Eq. (6):

$$
\frac{\partial V_{x}}{\partial x}+\frac{\partial V_{y}}{\partial y}+\frac{\partial V_{z}}{\partial z}=0
$$

In Eqs. (5) and (6), $V_{x}, V_{y}$, and $V_{z}$ are particle velocities along the $x, y$, and $z$ directions, respectively, and $D_{p}$ is the diffusion coefficient, which is proportional to particle size (Litwiniszyn 1963). The expression for the TCD is then obtained as in Eq. (7):

$Q_{t}=2 \pi D_{p} \exp \left(\frac{r_{0}^{2}}{4 D_{p} z_{0}}\right) z_{0}^{2}$

where, $Q_{t}$ is the total volume of the drawn out particles within a period $t$, and $z_{0}=z_{0}\left(r_{0}, t\right)$ are the particle coordinates when $t=0$. Considering that $D_{p}$ is related to the particle size in Eq. (7), the kinematic model can be used to investigate the effect of size of top coal on the shape of the TCD. When $r_{0}=0$, the maximum height of the TCD $\left(z_{0 \max }\right)$ according to Eq. (8) is

$Z_{0 \max }=\left(\frac{Q_{t}}{2 \pi D_{p}}\right)^{1 / 2}$

As is known from Eq. (8), when $z_{0 \max }$ is fixed, $Q_{t}$ increases linearly with $D_{p}$, which also increases linearly with the size of the top coal particles. Figure 4 shows the theoretical shapes of the TCD exposed to a fixed thickness of the coal seam and different sizes of top coal. 
2.3.1.3 Stochastic medium model Litwiniszyn (1956) first proposed stochastic medium theory to investigate the flow behavior of particles using probabilistic statistics. The model has been continually improved by scholars and introduced to block caving mining (Liu and Zhang 1995; Qiao et al. 2003). In recent years, it has also been applied to theoretical research on LTCC, and can adequately describe the shape of the TCD (Yu et al. 2017; Zhu et al. 2018). The assumptions are as follows:

(1) The blocks of top coal and gangue are completely granular blocks.

(2) Top coal and gangue can flow continuously, and are treated as a random medium.

(3) Their movement is independent in different directions.

Equations (9) and (10) are the probability density equations of top coal along the directions of advance and layout of the DS, respectively.

$P(x, z)=\frac{1}{A \sqrt{\pi \beta z^{\alpha}}} \exp \left\{-\frac{[x-f(z)]^{2}}{\beta z^{\alpha}}\right\}$

$P(y, z)=\frac{1}{\sqrt{\pi \beta_{1} z^{\alpha_{1}}}} \exp \left\{-\frac{y^{2}}{\beta_{1} z^{\alpha_{1}}}\right\}$

$A$ is average cutting coefficient of the rear canopy, and can be given by the following equations

$$
\begin{aligned}
& \int_{-\frac{z}{\tan \theta}}^{\infty} \frac{1}{A^{\prime} \sqrt{\pi \beta z^{\alpha}}} \exp \left\{-\frac{[x-f(Z)]^{2}}{\beta z^{\alpha}}\right\} \mathrm{d} x=1 \\
& A=\frac{\int_{0}^{H} \frac{1}{2} \operatorname{erf}\left\{-\frac{[Z+f(Z) \tan \theta]^{2}}{\sqrt{\beta z^{\alpha}}}\right\}+\frac{1}{2} \mathrm{~d} z}{H}
\end{aligned}
$$

The parameter $A$ in Eq. (11) is defined as the average cutting coefficient of the rear canopy, which can be calculated from the following method: it is assumed that the left particles moving boundary coincides with the extension line of the rear canopy, and $A^{\prime}$ ' in Eq. (11) is the cutting coefficient of the rear canopy. From the perspective of statistics, there is at least one particle in any layer to move down during top coal drawing. To reduce the calculation complexity and difficulty in solving the integral equation, $A^{\prime}$ is averaged along the vertical direction and $A$ is obtained. where $\theta$ is the dip angle of the rear canopy, $H$ is the height of the seam, $\alpha$ and $\beta$ are the coefficients along the direction of advance of the DS, and $\alpha_{1}$ and $\beta_{1}$ are coefficients along direction of layout of the DS. After combining Eqs. (9) and (11), the theoretical equation of the TCD along the direction of advance of the DS can be obtained in Eq. (12), where $z_{H}$ is the height of the drawnout top coal:

$\frac{[x-f(z)]^{2}}{\beta z^{\alpha}}=(\omega+1) \ln \frac{z_{H}}{Z}$

Figure 5 shows the theoretical shape of the TCD based on the stochastic medium model. The values of the parameters in Eq. 12 are $\alpha=1.54, \beta=0.34$. It is clear that the positions of the marked particles in physical testing (black points in Fig. 5) and in the theoretical equation (red fitted curved line in Fig. 5) are highly consistent, which indicates that the stochastic medium model can adequately fit the shape of the TCD when calibrated with the results of physical testing at the 2D scale.

For LTCC and blocking caving, the movements of top coal and gangue are both dominated by gravity, thus some basic models for blocking caving can be also applied to the LTCC. However, attention should be paid to due to the specific features of LTCC: (1) Drawing supports are arranged in LTCC panels, with an advancing process (2) Density of the drawn top coal is smaller than that of gangue
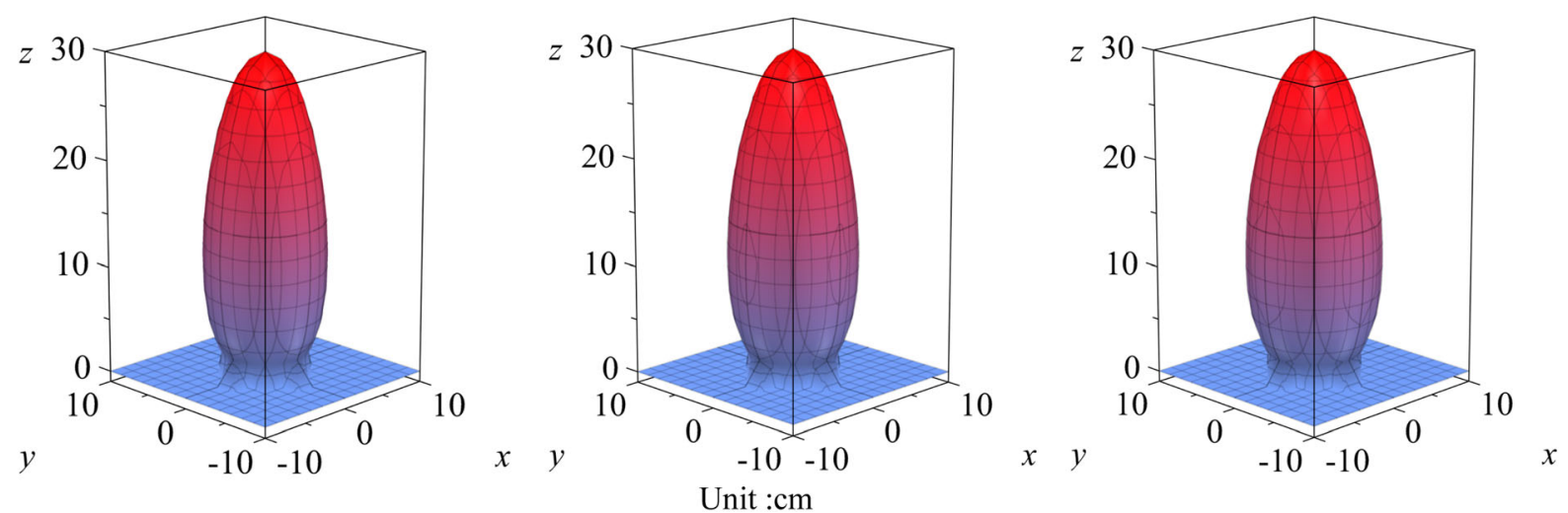

Fig. 4 TCD with different sizes of top coal: (a) $4.88 \mathrm{~mm}$, (b) $6.62 \mathrm{~mm}$ and (c) $8.30 \mathrm{~mm}$ 


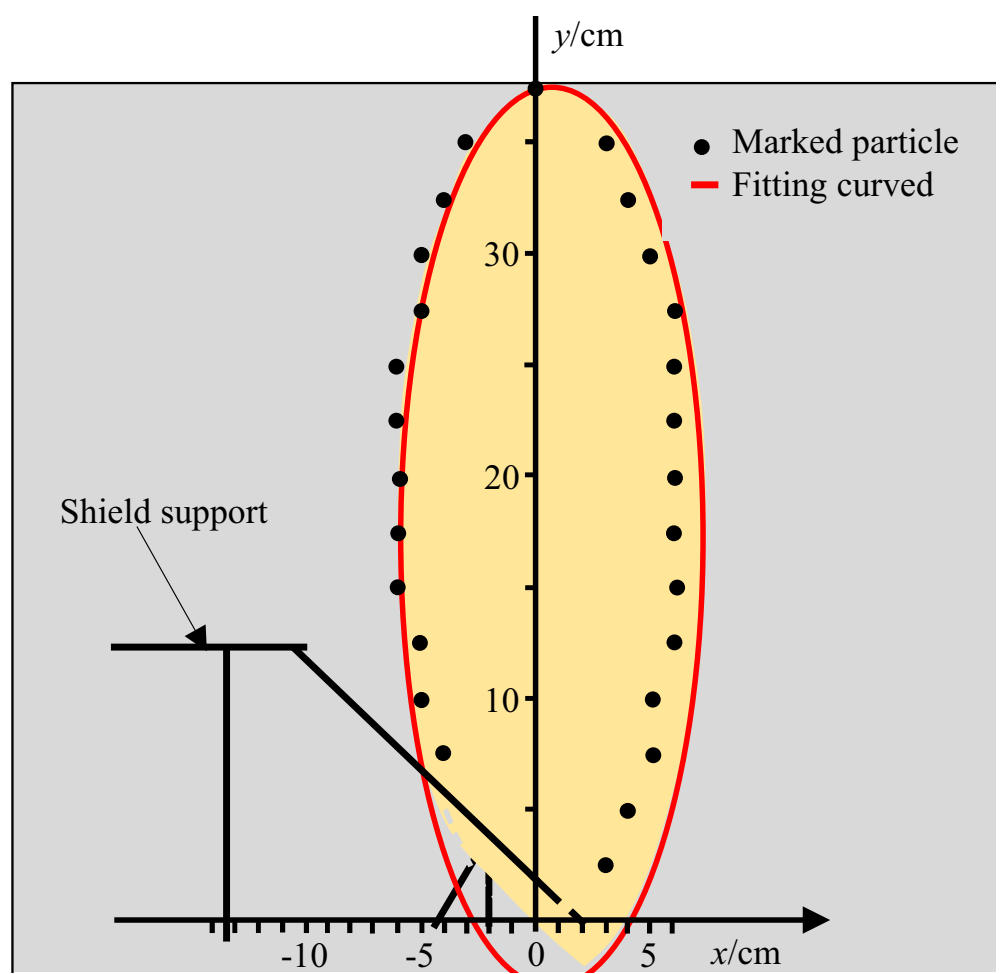

Fig. 5 Theoretical shape of the TCD based on stochastic medium model

in LTCC panels, while density of the ore block is much larger.

In summary, the B-R model, kinematic model and stochastic medium model are three commonly used models to characterize TCD. The B-R model mainly considers the force of the loose top coal particles, and then obtains the drawing body equation by calculating the moving path of the particles. For kinematic model, the state of top coal is regarded as the uninterruptible fluid, and the drawing body equation is obtained through the continuity equation. For stochastic medium model, the drawing body equation is calculated by considering the flowing probability of the particle. The three methods have distinctive advantages and disadvantages. In terms of the drawing body shape, it is wide for the top part and narrow for the bottom part in B-R model. However, it is opposite in kinematic model. In terms of application range, $\mathrm{B}-\mathrm{R}$ model is more suitable to establish the mechanical model.

\subsubsection{Models of TCB}

2.3.2.1 $2 \mathrm{D}$ model Based on stochastic medium theory, a 2D theoretical equation for the TCB can be obtained by analyzing the flow trajectory of the particles ( $\mathrm{Yu}$ et al. 2017; Zhu et al. 2018). The initial coordinates of a given particle are $\left(x_{0}, y_{0}\right)$, and the volume of the drawn out top coal is $S_{f}$ when the particle moves to a new location $(x, y)$. The flow trajectory of top coal and $S_{f}$ can be obtained by Eqs. (13) and (14), respectively, where $\alpha$ and $\beta$ are the coefficients along the direction of advance of the DS as documented in Eq. (11):

$\frac{x^{2}}{y^{\alpha}}=\frac{x_{0}^{2}}{y_{0}^{\alpha}}$

$S_{f}=\frac{2 A \sqrt{\pi \beta}}{\alpha+2}\left(y_{0}^{\frac{\alpha}{2}+1}-y^{\frac{\alpha}{2}+1}\right) \exp \left\{\frac{\left[x_{0}-f\left(y_{0}\right)\right]^{2}}{\beta y_{0}^{\alpha}}\right\}$

Combining Eqs. (13) and (14), the dynamic equation of the TCB can be obtained as shown in Eq. (15):

$\frac{[x-f(y)]^{2}}{\beta y^{\alpha}}=\ln \frac{(\alpha+2) S_{f}}{2 A \sqrt{\pi \beta}\left(y_{0}^{\frac{\alpha}{2}+1}-y^{\frac{\alpha}{2}+1}\right)}$

According to Eq. (15), the shape of the TCB can be obtained when subjected to different values of $S_{f}$ in the drawing process. When the first gangue flows out through the DO, the TCB is funnel shaped, and is also called the initial TCB. The theoretical equation of the initial TCB is

$\frac{[x-f(y)]^{2}}{\beta y^{\alpha}}=\ln \frac{H^{\frac{\alpha}{2}+1}}{H^{\frac{\alpha}{2}+1}-y^{\frac{\alpha}{2}+1}}$ 
As shown in Fig. 6, curves of various colors illustrate the theoretical shapes of the TCB subjected to different values of $S_{f}$. When the first drawing process is terminated, $S_{f}$ is $37.5 \mathrm{~m}^{2}$ and the shape of the TCB is indicated by the black curve in Fig. 6.

2.3.2.2 3D model Based on granular mechanics, the 3D equation of the TCB is deduced by analyzing the stress acting on the particles of top coal. As shown in Fig. 7, the force analysis includes the vertical force $\left(F_{N}\right)$, lateral force $(K \gamma H \Delta S)$, frictional force $(f)$, and the weight of the overlying gangue $(\gamma H \Delta S) \cdot \gamma$ is the unit weight, $\Delta S$ is the stressed area, $H$ is the thickness of the overlying gangue pillar, and $K$ is the lateral pressure coefficient.

When the drawing process is finished and the DO is closed, the particle reaches a balanced state as shown in Eq. (17):

$\left\{\begin{array}{c}-f \cos \theta+F_{N} \sin \theta=\gamma H \Delta S \\ f \sin \theta+F_{\text {lat }}=-F_{N} \cos \theta\end{array}\right.$

According to the Cartesian coordinate system in Fig. 7, Eq. (17) is transformed into Eq. (18).

$y^{\prime}=\tan \theta=\frac{m H \mu-1}{\mu+m H}$

where, $m$ is the transfer coefficient of lateral force and $\mu$ is the frictional coefficient between loose particles of top coal. Then, the equation of the TCB, Eq. (19), can be deduced through the integral of Eq. (18), where $M_{\mathrm{r}}$ and $M_{\mathrm{c}}$ are the thicknesses of the immediate roof and the coal seam, respectively:

$$
\begin{aligned}
y= & -\frac{\left(1+\mu^{2}\right)}{m} \ln (m H+\mu)+\mu H \\
& +\frac{1+\mu^{2}}{m} \ln \left(m\left(M_{r}+M_{c}\right)+\mu\right)-\mu\left(M_{r}+M_{c}\right)
\end{aligned}
$$

The 3D theoretical equation of the TCB can be derived by rotating Eq. (19):

$$
\begin{aligned}
y^{2}+z^{2}= & {\left[-\frac{\left(1+\mu^{2}\right)}{m}\right] \ln (m H+\mu)+\mu H } \\
& +\frac{\left(1+\mu^{2}\right)}{m} \ln \left(m\left(M_{r}+M_{c}\right)+\mu\right)-\mu\left(M_{r}+M_{c}\right)
\end{aligned}
$$

Figure 8a shows the theoretical 3D shape of the initial TCB, which fits the shape of the TCB in physical experiments by comparing the size and boundary of the DO. In addition, as shown in Fig. 8b, the theoretical equation of the TCB is also deduced when an excessive amount of gangue flows out, and the theoretical shape is different from the initial shape of the TCB such that it includes an elliptical top opening and rectangular bottom opening.

\section{Physical testing and numerical simulation}

\subsection{Brief summary of physical testing on drawing mechanism}

Table 2 summarizes the physical tests documented in the literature in last two decades, and classifies them based on the geometries of the relevant models (dimension, scaling, etc.) and the research items (such as the TCRR, TCD, and TCB were considered). The physical tests were often conducted to simulate multi-round top coal drawing

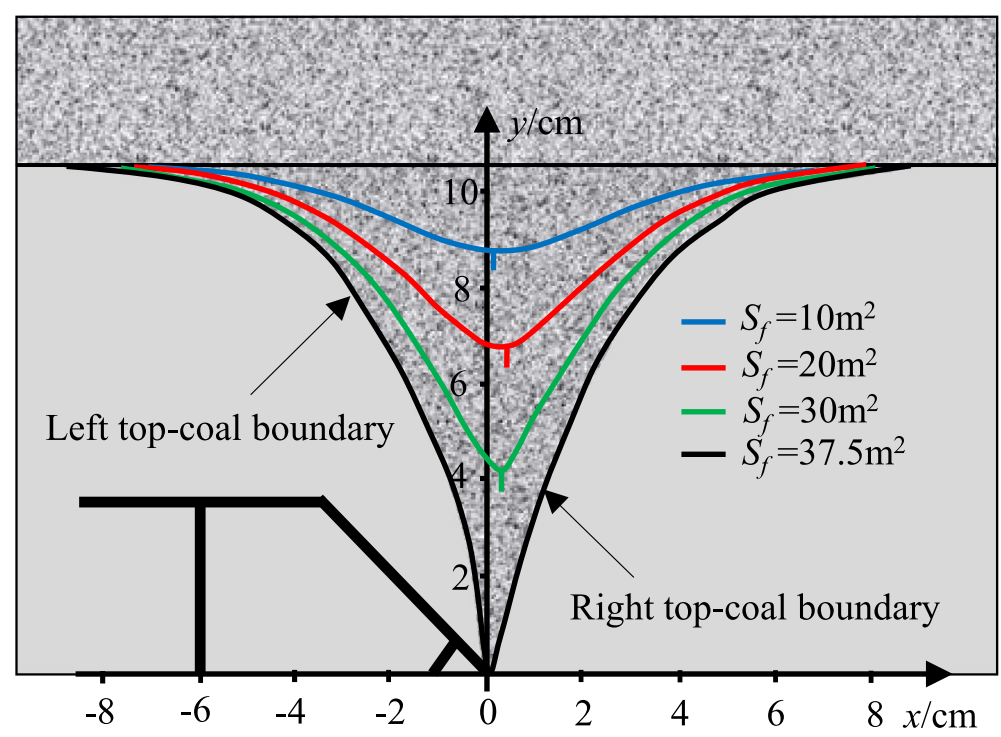

Fig. 6 Evolution of the TCB based on stochastic medium theory; after Zhu et al. (2018) 


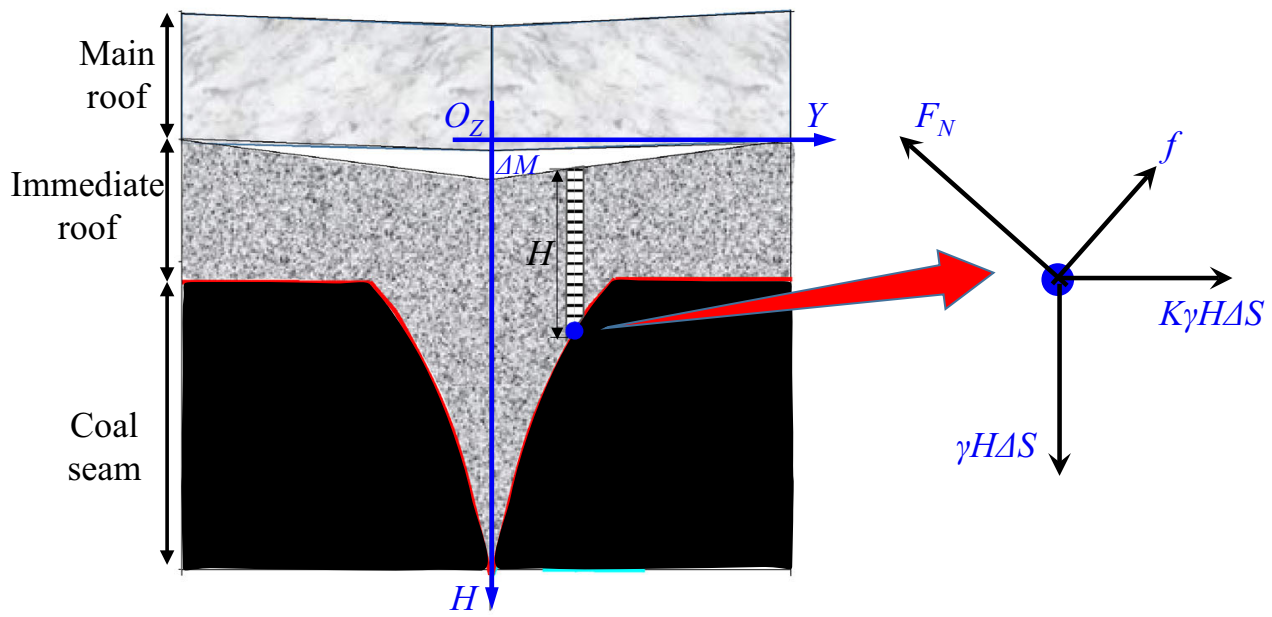

Fig. 7 Force analysis of top coal; after Wei et al. (2018)

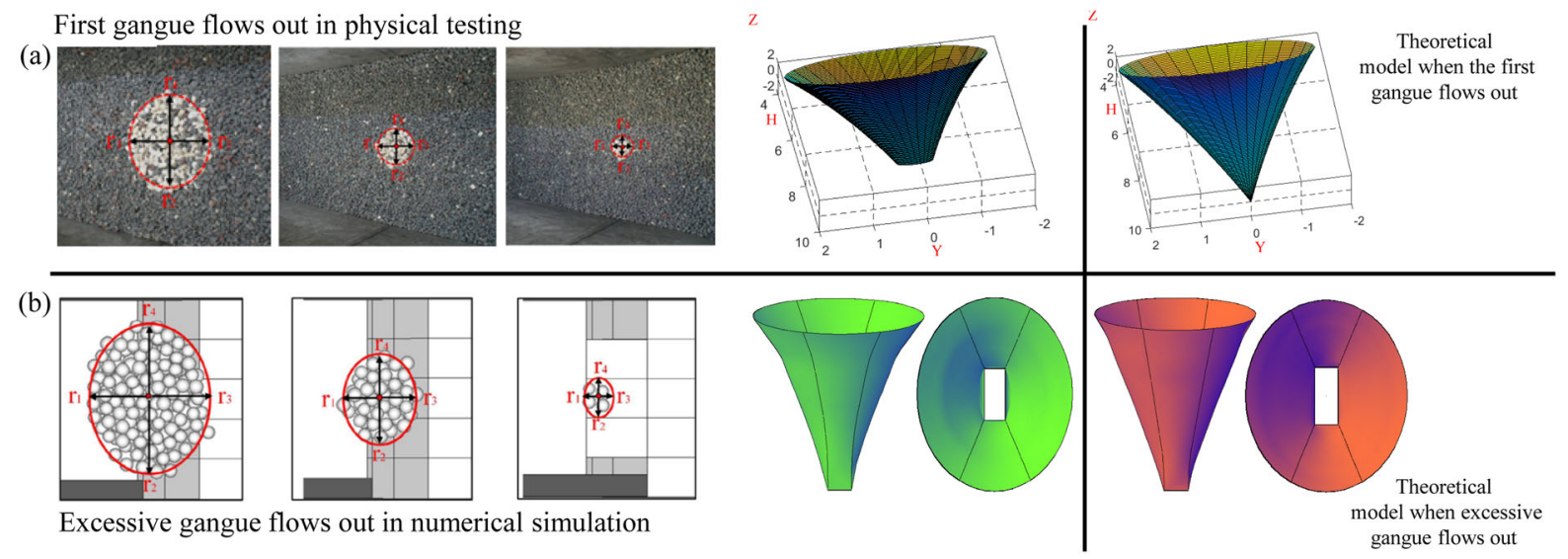

Fig. 8 Theoretical TCB: a first gangue flowing out in physical testing, b excessive gangue flowing out in numerical simulation

procedures. According to Table 2, a scaled ratio (usually varying between $1: 20$ and 1:40) is used to reduce the intensity of labor during the tests. Small rock fragments are often used to simulate the fractured top coal and gangue in lab testing (Huang et al. 2007; Wang et al. 2015a; Zhang et al. 2015a, 2018a), including limestone (Wang et al. 2019a), granite, and marble (Wang et al. 2015d, a). Their frictional coefficients are close to those of actual top coal and gangue in mines. Figure 9 shows typical devices used for top coal drawing tests (Wang et al. 2019b, a). A 3D testing device is shown in Fig. 9a, in which the DS can move forward as controlled by a screw to simulate the advancing support in mines. The corresponding results can be obtained and analyzed along the directions of advance and layout of the DS. A quasi-3D device is shown in Fig. 9b; the DS in this device cannot move forward due to restrictions along the direction of advance of the DS. Analysis is thus possible only along the direction of layout of the support.
"Marked particles" are often used in physical tests to characterize the shape of the TCD (Wang et al. 2015d, 2019a; Zhang et al. 2018a). Marked particles refer to numbered markers (the same materials as the top coal and gangue) that correspond to unique coordinate-related information to characterize location (Jin et al. 2017). Figure 10 illustrates some typical layouts of marked particles in physical tests (Wang et al. 2015d, 2019a; Zhang et al. 2018a). The spacing between marked particles directly impacts the accuracy of description of the TCD. The dense layout of the marked particles usually leads to a smooth boundary of the TCD. The TCD and TCRR can be calculated according to the location information and mass of the drawn out marked particles (Chen et al. 2017; Zhang et al. 2018a; Wang et al. 2019b, 2020b). Marked particles are also commonly used in block caving testing (Sharrock 2008; Jin et al. 2017). 
Table 2 Summary of experimental investigations of top coal drawing mechanism

\begin{tabular}{|c|c|c|c|c|c|c|c|c|c|}
\hline $\begin{array}{l}\text { Model } \\
\text { dimension }\end{array}$ & $\begin{array}{l}\text { Scaled } \\
\text { ratio }\end{array}$ & $\begin{array}{l}\text { Particle diameter } \\
(\mathrm{mm})\end{array}$ & $\begin{array}{l}\text { Support } \\
\text { number }\end{array}$ & $\begin{array}{l}\text { Support } \\
\text { advance }\end{array}$ & $\begin{array}{l}\text { Seam } \\
\text { angle }\left(^{\circ}\right)\end{array}$ & $\begin{array}{l}\text { TCRR } \\
\text { studied }\end{array}$ & $\begin{array}{l}\text { TCD } \\
\text { studied }\end{array}$ & $\begin{array}{l}\text { TCB } \\
\text { studied }\end{array}$ & Reference \\
\hline $3 \mathrm{D}$ & $1: 30$ & $2-20$ & 10 & $x$ & 0 & $x$ & $\sqrt{ }$ & $x$ & $\begin{array}{l}\text { Wang et al. } \\
(2015 \mathrm{c})\end{array}$ \\
\hline Quasi-3D & $1: 30$ & $5-30$ & 9 & $x$ & 80 & $\sqrt{ }$ & $\sqrt{ }$ & $\sqrt{ }$ & $\begin{array}{l}\text { Wang et al. } \\
\text { (2016d) }\end{array}$ \\
\hline Quasi-3D & $1: 30$ & $2-20$ & 1 & $\sqrt{ }$ & 0 & $x$ & $x$ & $\sqrt{ }$ & $\begin{array}{l}\text { Wang and Song } \\
\text { (2015) }\end{array}$ \\
\hline $3 \mathrm{D}$ & $1: 30$ & $5-12$ & 20 & $\sqrt{ }$ & 3 & $\sqrt{ }$ & $\sqrt{ }$ & $x$ & $\begin{array}{c}\text { Wang et al. } \\
\text { (2015a) }\end{array}$ \\
\hline $3 \mathrm{D}$ & $1: 30$ & $5-12$ & 20 & $\sqrt{ }$ & 22 & $\sqrt{ }$ & $\sqrt{ }$ & $\sqrt{ }$ & $\begin{array}{l}\text { Zhang et al. } \\
\text { (2015a) }\end{array}$ \\
\hline Quasi-3D & $1: 37.5$ & $4-26.7$ & 1 & $\sqrt{ }$ & 0 & $\sqrt{ }$ & $x$ & $\sqrt{ }$ & Liu et al. (2009) \\
\hline Quasi-3D & $1: 25$ & $5-17.5$ & 1 & $\sqrt{ }$ & 0 & $\sqrt{ }$ & $x$ & $\sqrt{ }$ & $\begin{array}{l}\text { Huang et al. } \\
\text { (2007) }\end{array}$ \\
\hline $3 \mathrm{D}$ & $1: 30$ & $1-15$ & 6 & $\sqrt{ }$ & 0 & $\sqrt{ }$ & $\sqrt{ }$ & $x$ & $\begin{array}{c}\text { Wang et al. } \\
\text { (2019a) }\end{array}$ \\
\hline $3 \mathrm{D}$ & $1: 30$ & $5-20$ & 20 & $\sqrt{ }$ & 45 & $\sqrt{ }$ & $\sqrt{ }$ & $\sqrt{ }$ & $\begin{array}{l}\text { Zhang et al. } \\
\text { (2018a) }\end{array}$ \\
\hline $3 \mathrm{D}$ & $1: 30$ & 6.62 & 6 & $\sqrt{ }$ & 0 & $\sqrt{ }$ & $\sqrt{ }$ & $x$ & $\begin{array}{c}\text { Wang et al. } \\
\text { (2020b) }\end{array}$ \\
\hline Quasi-3D & $1: 30$ & $5-12$ & 24 & $x$ & $0-50$ & $x$ & $\sqrt{ }$ & $x$ & $\begin{array}{l}\text { Wang et al. } \\
\text { (2019b) }\end{array}$ \\
\hline $3 \mathrm{D}$ & Unclear & $20-70$ & 3 & $x$ & 80 & $x$ & $x$ & $x$ & $\begin{array}{l}\text { Klishin et al. } \\
\text { (2013) }\end{array}$ \\
\hline Quasi-3D & $1: 37.5$ & $4-11.3$ & 1 & $\sqrt{ }$ & 0 & $\sqrt{ }$ & $x$ & $\sqrt{ }$ & Wang (2008) \\
\hline Quasi-3D & $1: 25$ & Unclear & 1 & $\sqrt{ }$ & 0 & $\sqrt{ }$ & $x$ & $\sqrt{ }$ & $\begin{array}{l}\text { Huang et al. } \\
\text { (2006) }\end{array}$ \\
\hline Quasi-3D & $1: 37.5$ & Unclear & 1 & $\sqrt{ }$ & 0 & $\sqrt{ }$ & $x$ & $\sqrt{ }$ & $\begin{array}{l}\text { Huang et al. } \\
\text { (2008) }\end{array}$ \\
\hline Quasi-3D & $1: 20$ & $10-20$ & 1 & $\sqrt{ }$ & 0 & $\sqrt{ }$ & $x$ & $x$ & $\begin{array}{l}\text { Wang et al. } \\
\text { (2014) }\end{array}$ \\
\hline
\end{tabular}

\subsection{Brief summary of numerical simulations of drawing mechanism}

Table 4 lists the numerical studies on top coal drawing mechanisms classified according to the software used, dimensions considered, and research items. Compared with the physical tests, the numerical methods exhibit specific advantages as well as defects. A comparison between the physical tests and numerical methods is presented in Table 3.

Figure 11 illustrates a numerical model of an LTCC panel. The rear canopy is modeled by considering the actual shape of the DS, and the DS can continuously move forward to simulate the advancement of the DS in the mine. Top coal can flow out of the DO when it is opened, and the thicknesses of the coal layers and gangue can be modeled by entering different parameters. The velocity of advancement of the DS as well as the drawing intervals can be assigned with different values according to the researcher's demands. For in-situ LTCC mines, the top coal above the rear canopy is fractured. When DO is opened, the top coal blocks can flow to the rear scraper conveyor. Therefore, the discrete methods, such as PFC and F/DEM, can be used to investigate the drawing mechanism. For the characteristics of roof movement and deformation in LTCC panels, the finite difference or finite element software, such as FLAC, is more convenient and applicable. In addition, the coupling of CDEM and bonded models makes it possible to analyze the top coal from the intact to fractured state.

\subsection{Classic conclusions based on physical testing and numerical simulations}

Physical tests and numerical simulations are both effective approaches to investigate the top coal drawing mechanism in LTCC. Based on them, some classic conclusions can be 

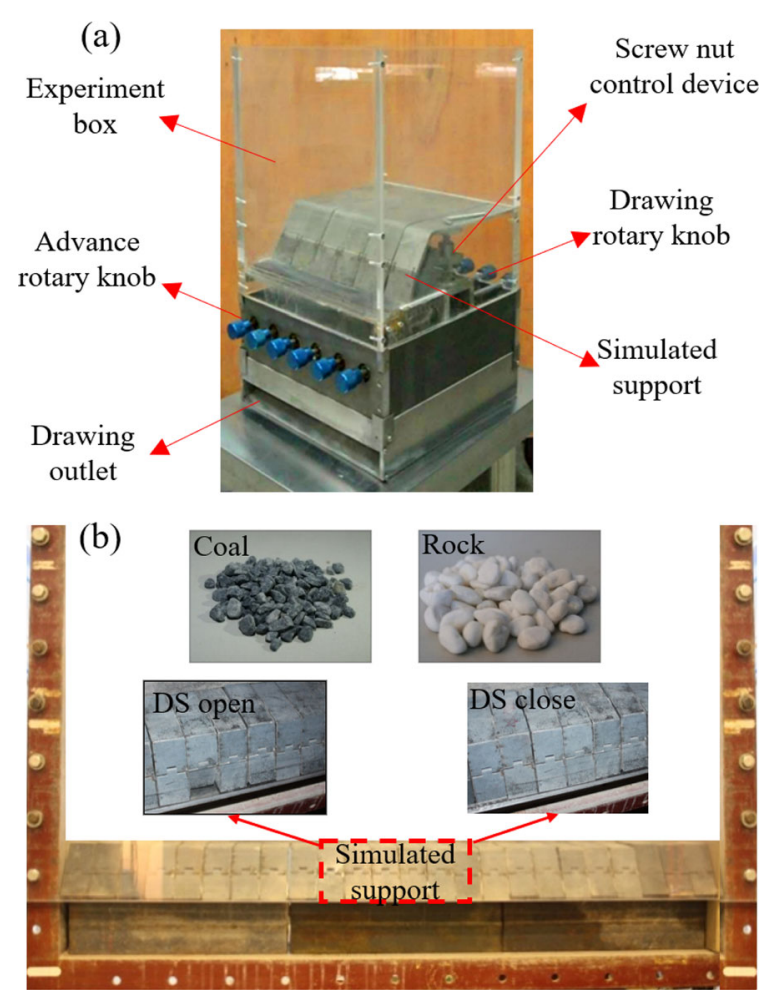

Fig. 9 A typical 3D testing device (Wang et al. 2019a) and a quasi-3D testing device (Wang et al. 2019b)
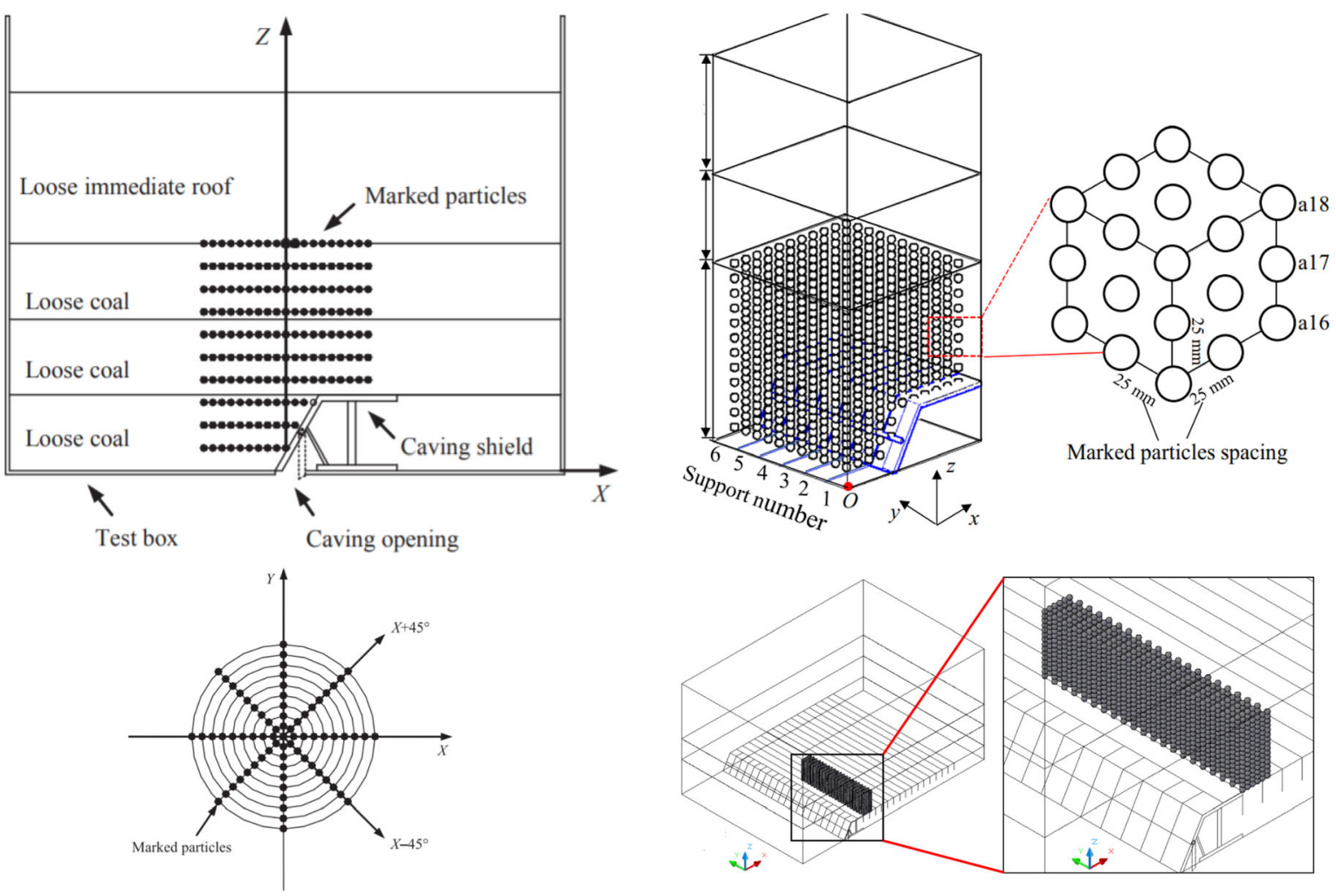

Fig. 10 Some layouts of marked particles in physical testing, reproduced from Wang et al. 2015c, 2019a; Zhang et al. 2018a 
Table 3 Comparisons between laboratory testing and PFC simulations, after Song and Konietzky (2019)

\begin{tabular}{|c|c|c|}
\hline Method & Physical test & Numerical simulation \\
\hline Advantages & $\begin{array}{l}\text { 1. Properties of materials used in physical testes are close to those } \\
\text { of coal and gangue fragments } \\
\text { 2. No need to calibrate the physical and mechanical parameters }\end{array}$ & $\begin{array}{l}\text { 1. Repeated testing is easy to conduct } \\
\text { 2. TCD boundary can be accurately visualized } \\
\text { 3. Flow path of a given top coal fragment can be traced and } \\
\text { shown } \\
\text { 4. Calculation of drawn out top coal (mass, ID, } \\
\text { coordinates) is accurate and automatic } \\
\text { 5. Coal loss and TCB are easily to visualize }\end{array}$ \\
\hline Disadvantages & $\begin{array}{l}\text { 1. Time consuming and labor intensive } \\
\text { 2. Repeated testing is time consuming } \\
\text { 3. TCB boundary is not easy to visualize accurately } \\
\text { 4. Flow path of top coal fragments cannot be visualized } \\
\text { 5. 3D TCB is not easy to visualize accurately } \\
\text { 6. Boundary of top coal cannot be visualized }\end{array}$ & $\begin{array}{l}\text { 1. Mechanical and physical parameters have to be } \\
\text { calibrated } \\
\text { 2. Non-spherical top coal fragments should be modeled; } \\
\text { spherical particles cause errors } \\
\text { 3. A large-scale simulation requires longer time and better } \\
\text { computer hardware }\end{array}$ \\
\hline
\end{tabular}

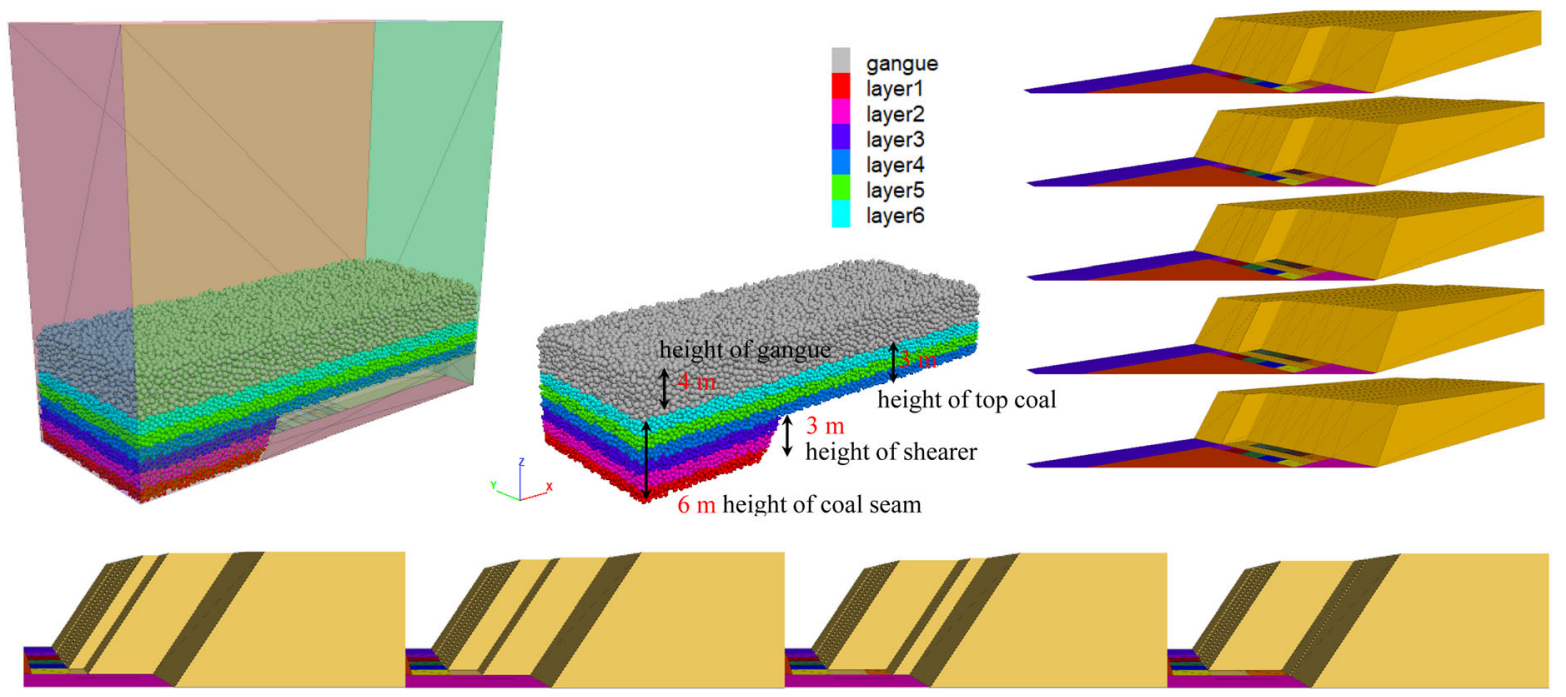

Fig. 11 Numerical modeling of an LTCC panel by considering the advance of the DS, after Song and Konietzky 2019

drawn. Some conclusions or mechanisms drawn from physical tests have also been validated by numerical simulations, and these are regarded as relatively reliable results. In this section, we summarize some classical conclusions that have been verified by both physical tests and numerical simulations.

\subsubsection{Conclusions on TCD}

\subsubsection{Effect of rear canopy of DS on TCD Figure 12} shows the effect of the rear canopy of the DS on the TCD based on experimental and simulation-based insights. As plotted in Fig. 12a, the shape of TCD is illustrated based on the drawn out marked particles in physical tests. The TCD without considering the effect of the DS (DS is not modeled) is approximately a symmetric ellipse (Wang et al. 2015d). This is similar to the shape of the IEZ in block caving mining (Melo et al. 2007; Jin et al. 2017; Song et al. 2018). When the effect of the DS is considered, it is clear that the TCD exhibits a deflection along the direction of advance of the support. To quantitatively characterize the deflection, a deflection angle, $\theta$, is proposed. It refers to the angle between the direction of the long axis of the ellipse and the plumb line, as shown in Fig. 12a. It shows that the extent of the deflection $(\theta)$ decreases with an increase in the height of the TCD (Wang et al. 2015d), which indicates that the effect of the DS is more pronounced when the height of the TCD is small, and the effect of the deflection is weakened when the height is much greater than the height of the DS. A similar 
Table 4 Summary of numerical simulations on the drawing mechanism in LTCC

\begin{tabular}{|c|c|c|c|c|c|c|c|c|c|}
\hline $\begin{array}{l}\text { Model } \\
\text { type }\end{array}$ & $\begin{array}{l}\text { Particle } \\
\text { shape }\end{array}$ & Software & $\begin{array}{l}\text { Support } \\
\text { number }\end{array}$ & $\begin{array}{l}\text { Support } \\
\text { advance }\end{array}$ & $\begin{array}{l}\text { Seam angle } \\
\left({ }^{\circ}\right)\end{array}$ & $\begin{array}{l}\text { TCRR } \\
\text { studied }\end{array}$ & $\begin{array}{l}\text { TCD } \\
\text { studied }\end{array}$ & $\begin{array}{l}\text { TCB } \\
\text { studied }\end{array}$ & Reference \\
\hline $3 \mathrm{D}$ & Sphere & $\mathrm{PFC}^{3 \mathrm{D}}$ & 7 & $\sqrt{ }$ & 0 & $\sqrt{ }$ & $\sqrt{ }$ & $\sqrt{ }$ & $\begin{array}{l}\text { Song and Konietzky } \\
\text { (2019) }\end{array}$ \\
\hline $3 \mathrm{D}$ & Sphere & $\mathrm{PFC}^{3 \mathrm{D}}$ & 7 & $\sqrt{ }$ & 0 & $\sqrt{ }$ & $\sqrt{ }$ & $\sqrt{ }$ & Song et al. (2020) \\
\hline $2 / 3 \mathrm{D}$ & Polytope & $\mathrm{PFC}^{2 / 3 \mathrm{D}}$ & 1 & $x$ & 0 & $\sqrt{ }$ & $\sqrt{ }$ & $\sqrt{ }$ & Song et al. (2018) \\
\hline $2 \mathrm{D}$ & Disk & $\mathrm{PFC}^{2 \mathrm{D}}$ & 19 & $x$ & 80 & $\sqrt{ }$ & $x$ & $\sqrt{ }$ & Wang et al. (2016d) \\
\hline $2 \mathrm{D}$ & Disk & $\mathrm{PFC}^{2 \mathrm{D}}$ & 1 & $\sqrt{ }$ & 0 & $\sqrt{ }$ & $x$ & $\sqrt{ }$ & Wang et al. (2014) \\
\hline $3 \mathrm{D}$ & Sphere & $\mathrm{PFC}^{3 \mathrm{D}}$ & 20 & $x$ & 45 & $\sqrt{ }$ & $\sqrt{ }$ & $\sqrt{ }$ & Zhang et al. (2018a) \\
\hline $2 \mathrm{D}$ & Disk & $\mathrm{PFC}^{2 \mathrm{D}}$ & 1 & $\sqrt{ }$ & $0-40$ & $\sqrt{ }$ & $x$ & $\sqrt{ }$ & Yang et al. (2016) \\
\hline $2 \mathrm{D}$ & Disk & CDEM & 1 & $\sqrt{ }$ & 0 & $\sqrt{ }$ & $x$ & $x$ & Zhang et al. (2019) \\
\hline $2 \mathrm{D}$ & Disk & $\mathrm{PFC}^{2 \mathrm{D}}$ & 10 & $x$ & $0-50$ & $x$ & $\sqrt{ }$ & $\sqrt{ }$ & Wang et al. (2019b) \\
\hline $3 \mathrm{D}$ & Sphere & $\mathrm{PFC}^{3 \mathrm{D}}$ & 5 & $x$ & 0 & $\sqrt{ }$ & $\sqrt{ }$ & $\sqrt{ }$ & Wang et al. (2013) \\
\hline $3 \mathrm{D}$ & Sphere & $\mathrm{PFC}^{3 \mathrm{D}}$ & 5 & $x$ & 0 & $\sqrt{ }$ & $\sqrt{ }$ & $\sqrt{ }$ & $\begin{array}{l}\text { Wang and Zhang } \\
\quad(2015)\end{array}$ \\
\hline $2 \mathrm{D}$ & Disk & $\mathrm{PFC}^{2 \mathrm{D}}$ & 20 & $x$ & 0 & $\sqrt{ }$ & $\sqrt{ }$ & $x$ & Wang et al. (2020b) \\
\hline $3 \mathrm{D}$ & Sphere & $\mathrm{PFC}^{3 \mathrm{D}}$ & 5 & $x$ & 0 & $x$ & $\sqrt{ }$ & $x$ & Wang et al. (2015c) \\
\hline $2 \mathrm{D}$ & Disk & $\mathrm{PFC}^{2 \mathrm{D}}$ & 1 & $\sqrt{ }$ & 0 & $\sqrt{ }$ & $\sqrt{ }$ & $\sqrt{ }$ & Zhang et al. (2016) \\
\hline $2 \mathrm{D}$ & Disk & $\mathrm{PFC}^{2 \mathrm{D}}$ & 1 & $\sqrt{ }$ & 0 & $x$ & $x$ & $\sqrt{ }$ & Zhang et al. (2015b) \\
\hline $2 \mathrm{D}$ & Disk & F/DEM & 1 & $\sqrt{ }$ & 0 & $x$ & $\sqrt{ }$ & $\sqrt{ }$ & Huo et al. (2020) \\
\hline $3 \mathrm{D}$ & Sphere & $\mathrm{PFC}^{3 \mathrm{D}}$ & 5 & $x$ & 0 & $x$ & $x$ & $\sqrt{ }$ & Wei et al. (2018) \\
\hline $2 \mathrm{D}$ & Disk & $\mathrm{PFC}^{2 \mathrm{D}}$ & 1 & $x$ & 0 & $x$ & $x$ & $x$ & Xie and Zhao (2009a) \\
\hline $2 \mathrm{D}$ & Disk & $\mathrm{PFC}^{2 \mathrm{D}}$ & Unclear & $x$ & 64 & $x$ & $x$ & $x$ & Miao et al. (2011) \\
\hline $3 \mathrm{D}$ & Block & FLAC $^{3 \mathrm{D}}$ & Unclear & $x$ & 0 & $x$ & $\sqrt{ }$ & $x$ & $\begin{array}{l}\text { Yasitli and Unver } \\
\quad(2005)\end{array}$ \\
\hline $3 \mathrm{D}$ & Disk & $\mathrm{PFC}^{2 \mathrm{D}}$ & 20 & $x$ & 0 & $\sqrt{ }$ & $\sqrt{ }$ & $\sqrt{ }$ & Wang et al. (2020a) \\
\hline $3 \mathrm{D}$ & Sphere & $\mathrm{PFC}^{3 \mathrm{D}}$ & 20 & $x$ & $0-50$ & $\sqrt{ }$ & $\sqrt{ }$ & $\sqrt{ }$ & Zhang et al. (2018b) \\
\hline
\end{tabular}

phenomenon is observed in Fig. 12b based on the results of a numerical simulation. With an increase in the height of the TCD, the deflection in it decreases. The simulation shows that the TCD shows a specific "cut-variant ellipsoid" shape when the effect of the rear canopy of the DS is considered (Wang and Zhang 2015; Wang et al. 2016d, b). Figure $12 \mathrm{~b}$ also compares the extent of deflection of the TCD between the approaches, and shows that the deflection in both physical tests and numerical simulations exhibits similar behaviors with regard to TCD height ( $\mathrm{z}$ coordinate). It is concluded that the rear canopy of the DS causes deflections in the TCD. Figure 12c shows the effect of the angle of the rear canopy on the TCD (Song et al. 2020). As the angle increases from $45^{\circ}$ to $72^{\circ}$, the deflection of the TCD is weakened. It can be concluded that a smaller value of the angle of the rear canopy corresponds to a more pronounced deflection of the TCD.

3.3.1.2 Effect of coal seam inclination on TCD Figure 13 illustrates the shapes of the TCD subjected to different dip angles of the coal seams $\left(0-50^{\circ}\right)$ based on physical testing and numerical simulations (Zhang et al. 2018b, a; Wang et al. 2019b).

Figure 13a shows the effect of the dip angle on the TCD in physical tests, and the results of the corresponding numerical simulation are shown in Fig. 13b. It is clear that the TCD exhibits a similar pattern in both approaches. With an increase in the dip angle, the TCD exhibits a more pronounced deflection toward the upper side of the face of the panel. In addition, the quality (volume) of the TCD increases with the rise in seam inclination. Figure 14 summarizes the effect of the dip angle on TCD quality (volume) based on the results documented by Yang et al. (2016), Zhang et al. (2018b) and Wang et al. (2019b). It reveals that with the rise in the dip angle (at least within the range of $0-50^{\circ}$ ), the quality or volume of the TCD exhibits an approximately linear increase. One interpretation of the increased TCD quality when subjected to an inclined seam is that the inclination of the coal seam its thickness as well as the drawing height of the TCD. 

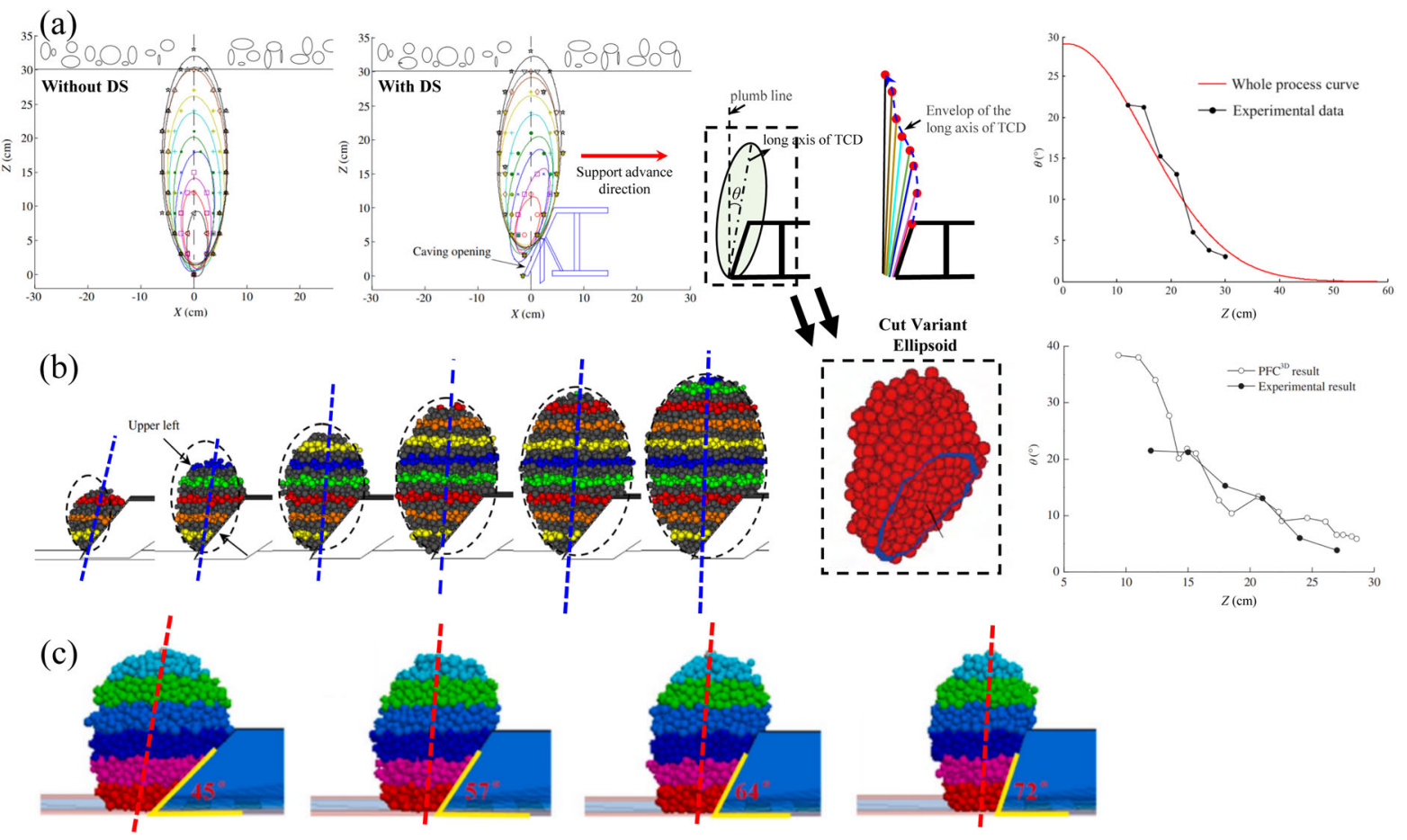

Fig. 12 a Deflections in the TCD in physical tests. b Deflections in the TCD in simulations, after Wang et al. (2015c). c Effect of rear canopy on the TCD, reproduced from Song et al. (2020)

3.3.1.3 Effect of sizes of top coal and DO on TCD Many studies have reported that the sizes of top coal and DO have a significant effect on the shape of the TCD (Wang et al. 2016c, 2019a, 2020a). Table 5 shows the detailed size distribution of top coal in three schemes in a physical test. The percentage of top coal samples of small size is high in scheme 2 but low Melo 3, which indicates that the average size of top coal is the smallest in scheme 2 and the largest in scheme 3. In addition, the variation in the size of the DO changes the relative size of top coal (size of top coal divided by the size of DO), resulting in the different movements of top coal (Zhang et al. 2018a; Wang et al. 2020b). Figure 15 illustrates the shape of the TCD subjected to three different sizes of top coal and DO. By analyzing the number and position of the marked particles drawn from different DS, the number of drawn out marked
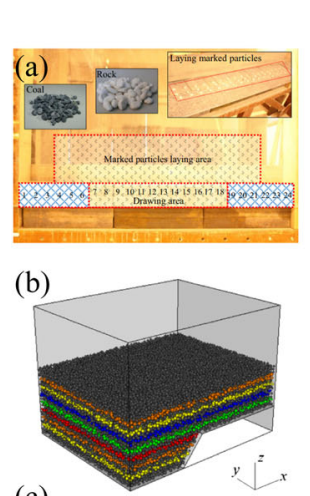

(c)

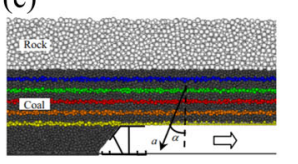

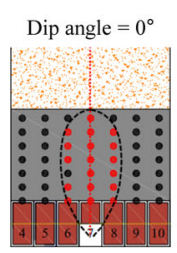
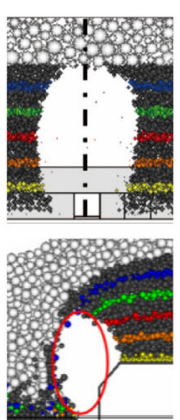
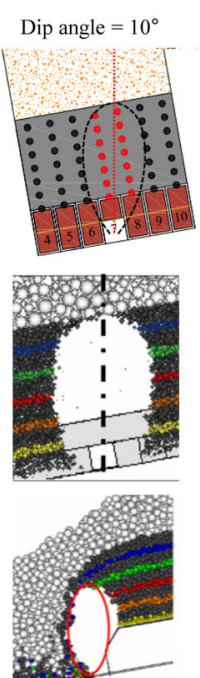
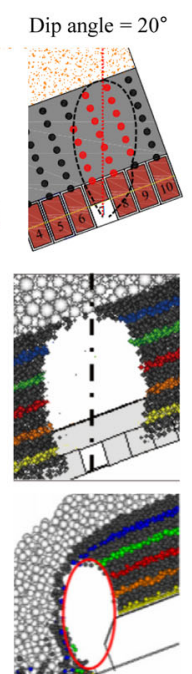
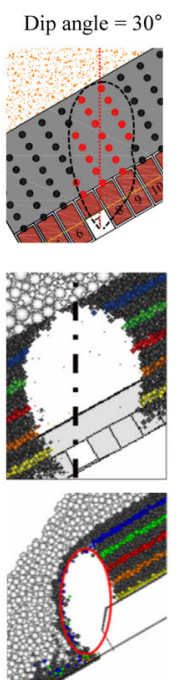
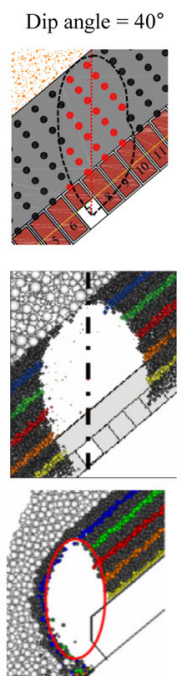
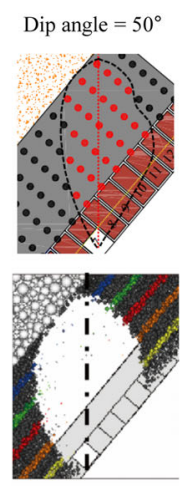

Fig. 13 Effect of dip angle on the TCD: a lab tests results, after Wang et al. (2019b); b numerical simulation: direction of layout of the DS; c numerical simulation: direction of advance of the DS, after Yang et al. (2016) 


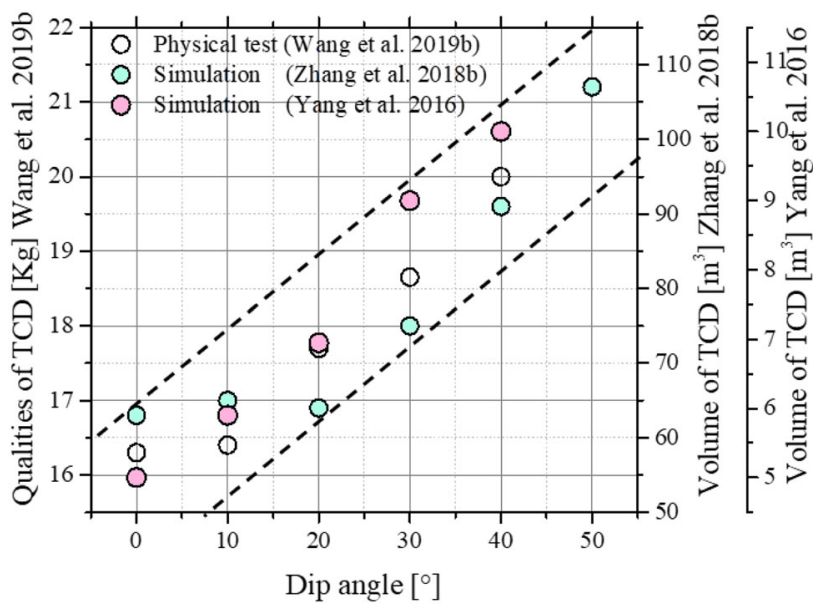

Fig. 14 TCD quality (volume) versus dip angle

particles in the right side is larger than that in the left side. This is owing to smoother contact between the top coal and the DS, which causes the TCD to develop toward the support side. It can be concluded that when the size of the DO is fixed, the volume of the TCD linearly increases with the growth in the size of top coal. When the latter is fixed, the ratio of width to height of the TCD gradually increases with the size of the DO, which indicates that the TCD is fatter.

\subsubsection{Conclusions on TCRR}

The Top coal recovery ratio (TCRR) is an important economic indicator to evaluate the production efficiency of an LTCC mine (Wang et al. 2015b; Zhu et al. 2018). A low TCRR often results in the loss of a large amount of permanent coal. Many scholars have investigated methods to improve the TCRR by using lab testing and numerical simulations (Xie and Zhao 2009b; Wang et al. 2016d; Yang et al. 2016; Zhang and Wimmer 2018; Zhu et al. 2018; Zhang et al. 2019). This section focuses on the effects of the drawing parameters (such as drawing interval, drawing sequence, top coal size, dip angle of coal seam, etc.) on the TCRR,
3.3.2.1 Effect of drawing sequence on TCRR Horizontal coal seam The drawing sequence has a direct impact on the TCRR in a horizontal coal seam (Wang et al. 2016a, c). Table 6 presents the TCRR in the horizontal coal seam subjected to different drawing sequences, investigated using both physical tests and numerical simulations. Nine schemes were used, and the corresponding results of the TCRR are shown in Table 6. Schemes 1-3 considered five DS in a physical test. The results show that the sequential drawing (scheme 1) had the lowest TCRR (80.7\%). Schemes 2 and 3 used the alternate drawing, and one with the largest interval (scheme 3) had the largest TCRR. Schemes 4-9 studied the effects of the DO size (number of DO used in drawing) on the TCRR by performing experiments and numerical simulations. They concluded that the TCRR increases with DO size. To sum up, alternate and multi-DO drawings are beneficial for increasing the TCRR.

Inclined coal seam Figure 16 illustrates the effects of the drawing sequence on the TCRR in a steeply inclined (around $80^{\circ}$ ) coal seam (Wang et al. 2016d). Two drawing sequences were used:

(1) roof-floor drawing, shown in Fig. 16a;

(2) floor-roof drawing, shown in Fig. 16b.

The losses in coal for the two plans are clearly distinct. The roof-floor drawing led to a clearly higher loss, which corresponded to a smaller TCRR than the floor-roof drawing. The simulation in Fig. 16c also confirms this conclusion. This results hint that for a steeply inclined seam, the floor-roof drawing is preferable for a higher TCRR.

3.3.2.2 Effects of drawing interval on TCRR Figure 17 summarizes the effects of the drawing interval on the TCRR. The drawing procedures used on site are usually of three types:

(1) Top coal drawing is performed after one-time support advance.

(2) Top coal drawing is performed after two-time support advance.

(3) Top coal drawing is performed after three-time support advance.

Table 5 Size distribution of top coal in different schemes

\begin{tabular}{llrrrrr}
\hline Particle diameter & $0-1 \mathrm{~mm}$ & $1-2 \mathrm{~mm}$ & $2-3 \mathrm{~mm}$ & $3-5 \mathrm{~mm}$ & $5-10 \mathrm{~mm}$ & $10-15 \mathrm{~mm}$ \\
\hline Mass & Mass percentage $(\%)$ & & & \\
Scheme 1 & 14.76 & 8.61 & 8.05 & 13.02 & 25.00 & 30.56 \\
Scheme 2 & 14.65 & 17.93 & 17.55 & 12.63 & 21.25 & 16.00 \\
Scheme 3 & 13.06 & 2.01 & 3.02 & 8.05 & 27.18 \\
\hline
\end{tabular}




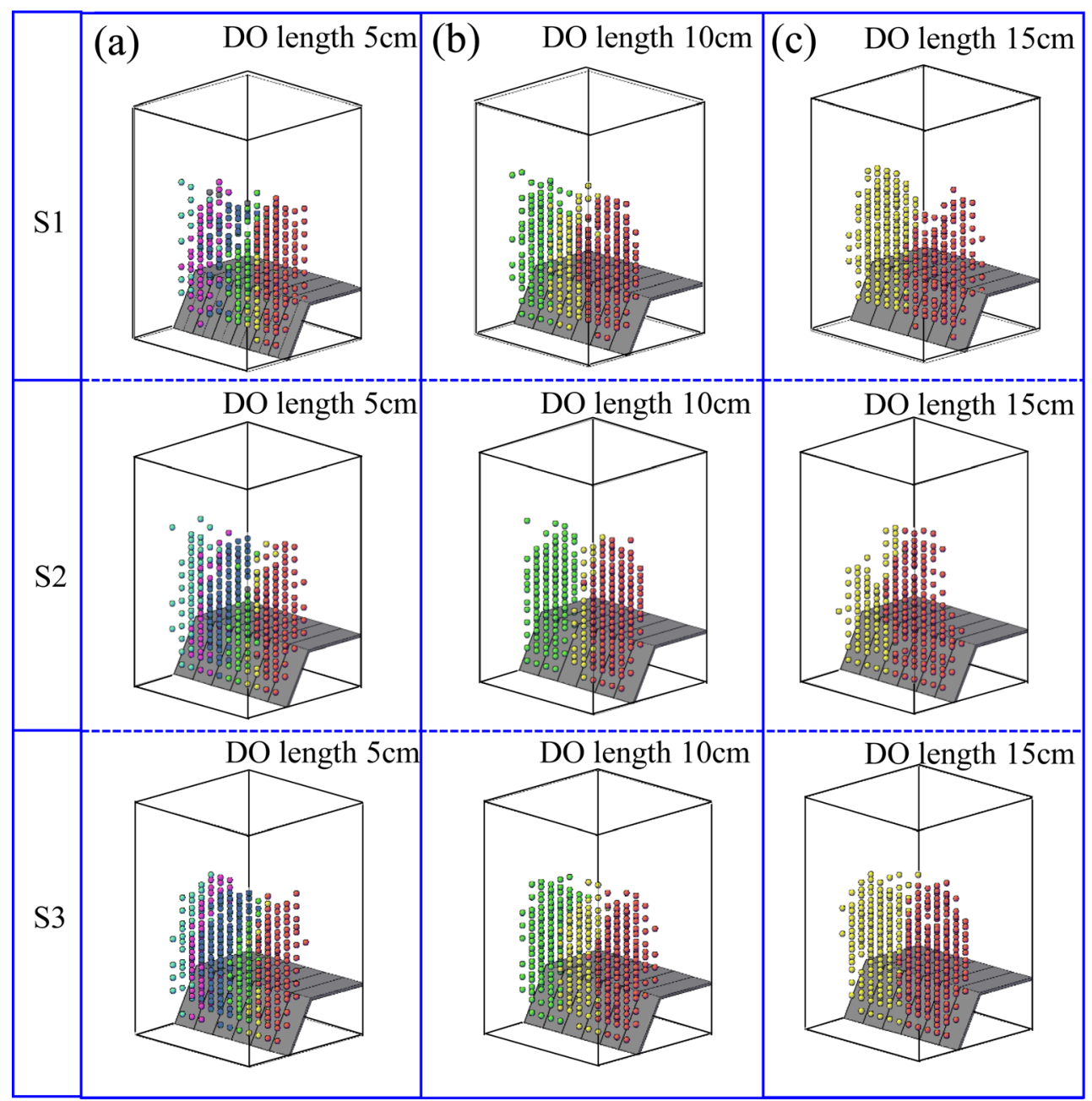

Fig. 15 Effects of top coal and DO size on the TCD: a DO size, $5 \mathrm{~cm}$; b DO size, $10 \mathrm{~cm}$; c DO size, $15 \mathrm{~cm}$; after Wang et al. (2019a)

Table 6 Effects of drawing sequence on the TCRR

\begin{tabular}{llllll}
\hline Plan & Drawing sequence & Method & DO used & TCRR & DS layout \\
\hline 1 & {$[1]-[2]-[3]-[4]-[5]$} & Physical tests & 1 & $80.7 \%$ & Physical tests \\
2 & {$[1]-[3]-[5]-[2]-[4]$} & 1 & $87.4 \%$ \\
3 & {$[1]-[5]-[3]-[2]-[4]$} & Physical tests & 1 & $95.4 \%$ \\
4 & {$[1]-[2]-[3]-[4]-[5]-[6]-[7]-[8]-[9]-[10]-[11]-[12]$} & Simulation & 1 & $77.59 \%$ \\
5 & {$[1-2]-[3-4]-[5-6]-[7-8]-[9-10]-[11-12]$} & Simulation & 2 & $80.32 \%$ \\
6 & {$[1-3]-[4-6]-[7-9]-[10-12]$} & Simulation & 3 & $81.07 \%$ \\
7 & {$[1]-[2]-[3]-[4]-[5]-[6]-[7]-[8]-[9]-[10]-[11]-[12]$} & Physical tests & 1 & $70.24 \%$ \\
8 & {$[1-2]-[3-4]-[5-6]-[7-8]-[9-10]-[11-12]$} & Physical tests & 2 & $78.57 \%$ \\
9 & {$[1-3]-[4-6]-[7-9]-[10-12]$} & Physical tests & 3 & $89.68 \%$ & $\square \square \square \square \square$ \\
\hline
\end{tabular}

Figure 17 presents the TCRR versus different drawing internals at a lab testing scale based on the published literature. Some studies have conclude that "drawing after two-time support advance" leads to the largest TCRR (Huang et al. 2006, 2008; Wang et al. 2014). However, other studies have obtained different results (Wang 2008). As shown in Fig. 17, data from Wang (2008) shows that "drawing after two-time support advance" does not yield the largest TCRR in most cases, and "drawing after onetime support advance" usually leads to the largest TCRR. 
(a) Roof-Floor
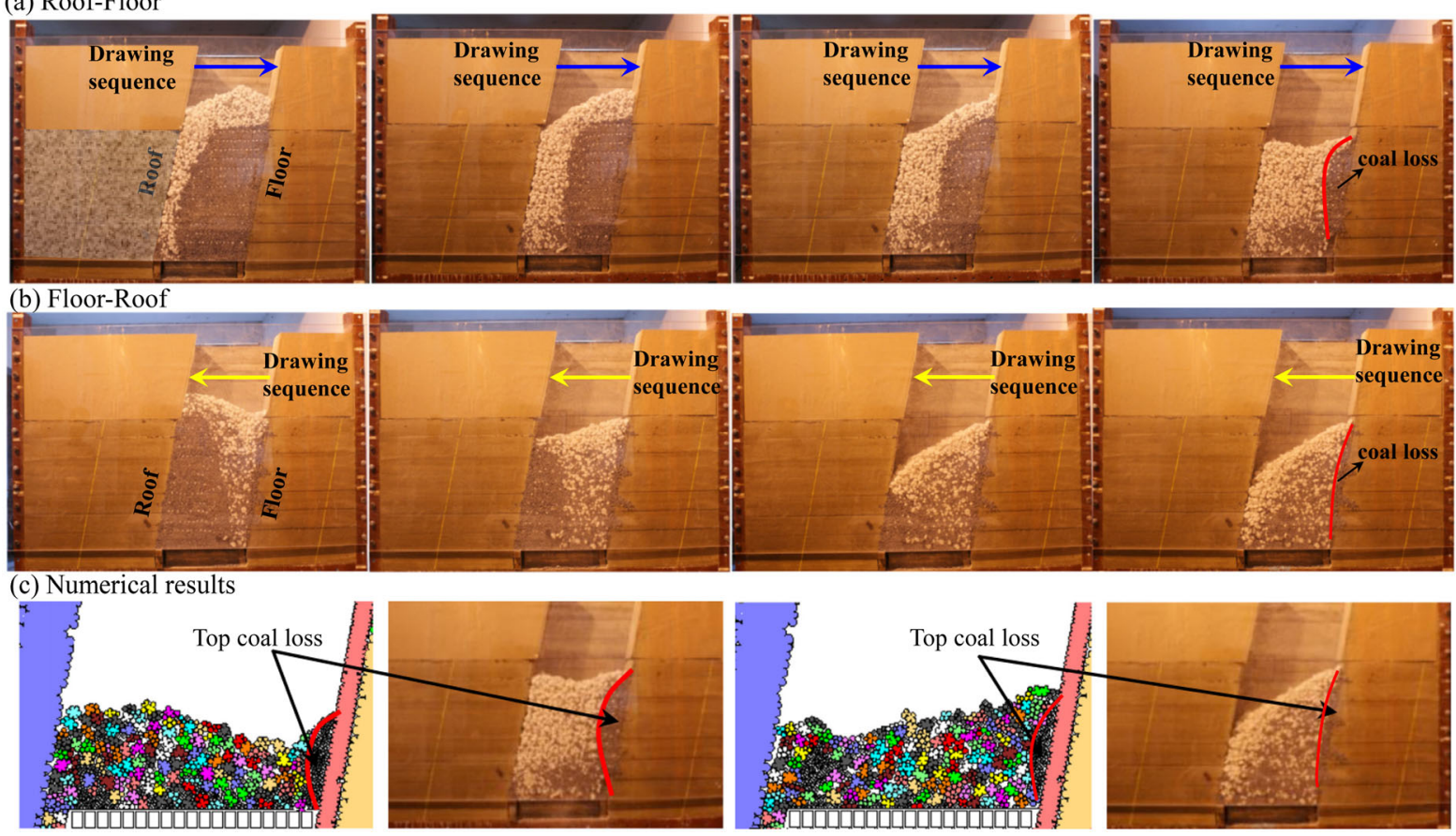

Fig. 16 a Roof-floor drawing; b floor-roof drawing; c results of simulations, after Wang et al. (2016d)

The same study (Wang 2008) also examined the TCRR when subjected to distinct cutting-drawing ratios (C/D ratio: the ratio of bottom coal/top coal thickness), which indicates that the impact of the drawing interval on the TCRR is also influenced by other parameters such as the C/D ratio. However, it can least be concluded that "drawing after three-time support advance" always yields the smallest TCRR. To sum up, a larger drawing interval is not recommended as it may cause a smaller TCRR.

\subsubsection{Conclusions on TCFB}

3.3.3.1 Flow trajectory The flow trajectory can be precisely visualized only with the aid of a numerical simulation. Physical testing cannot trace the flow trajectory of a given particle during the drawing process. Figure 18 presents the trajectories of particles of top coal in a numerical simulation. Figure 18a shows three trajectories of different particles of top coal (Zhang et al. 2018a), where flow traces
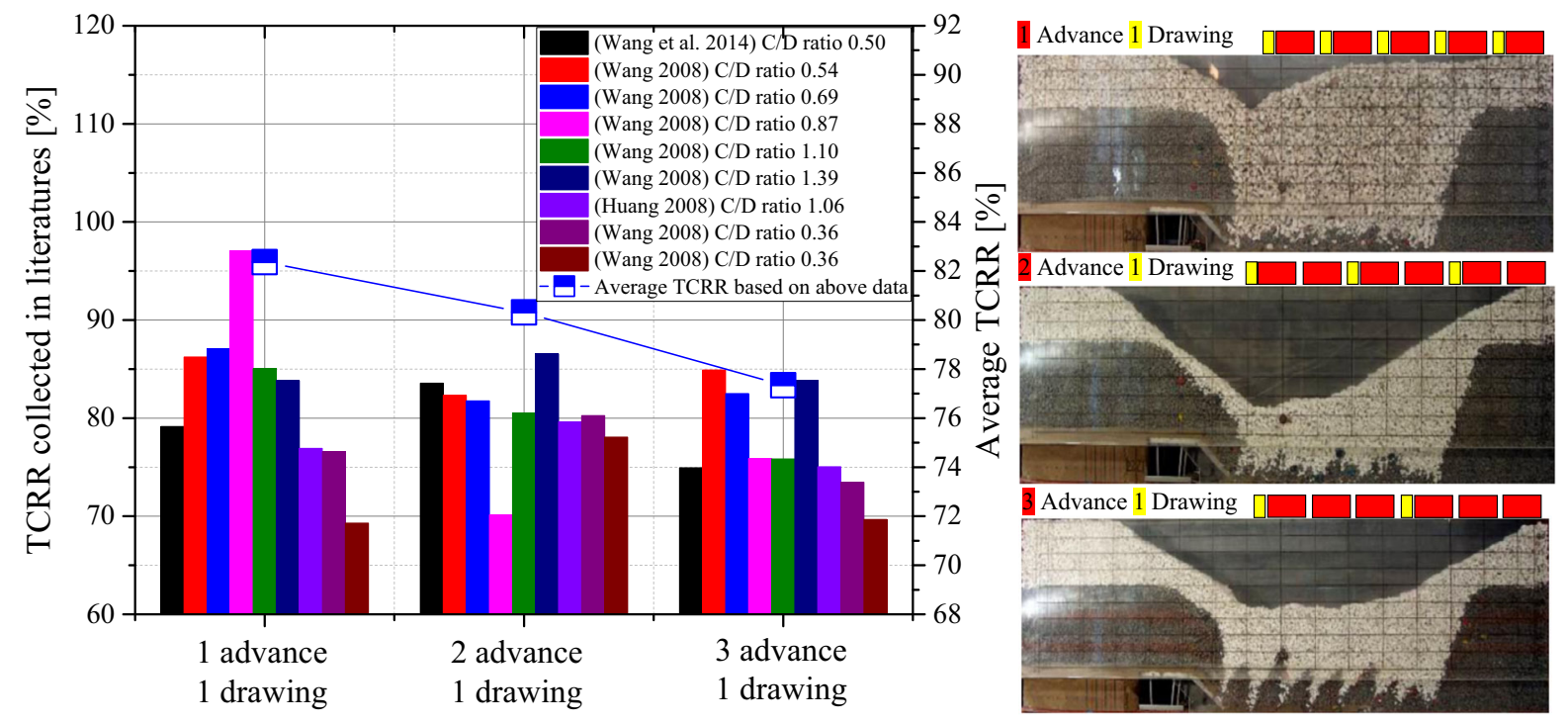

Fig. 17 Effect of drawing interval on TCRR 
(a)

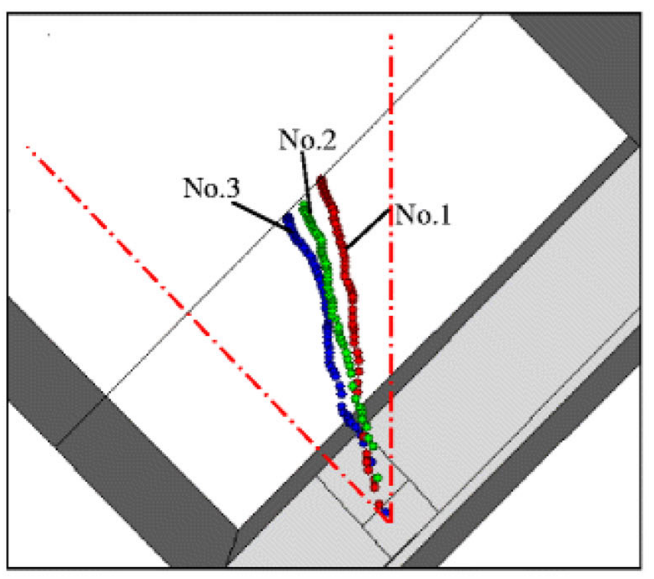

(b)
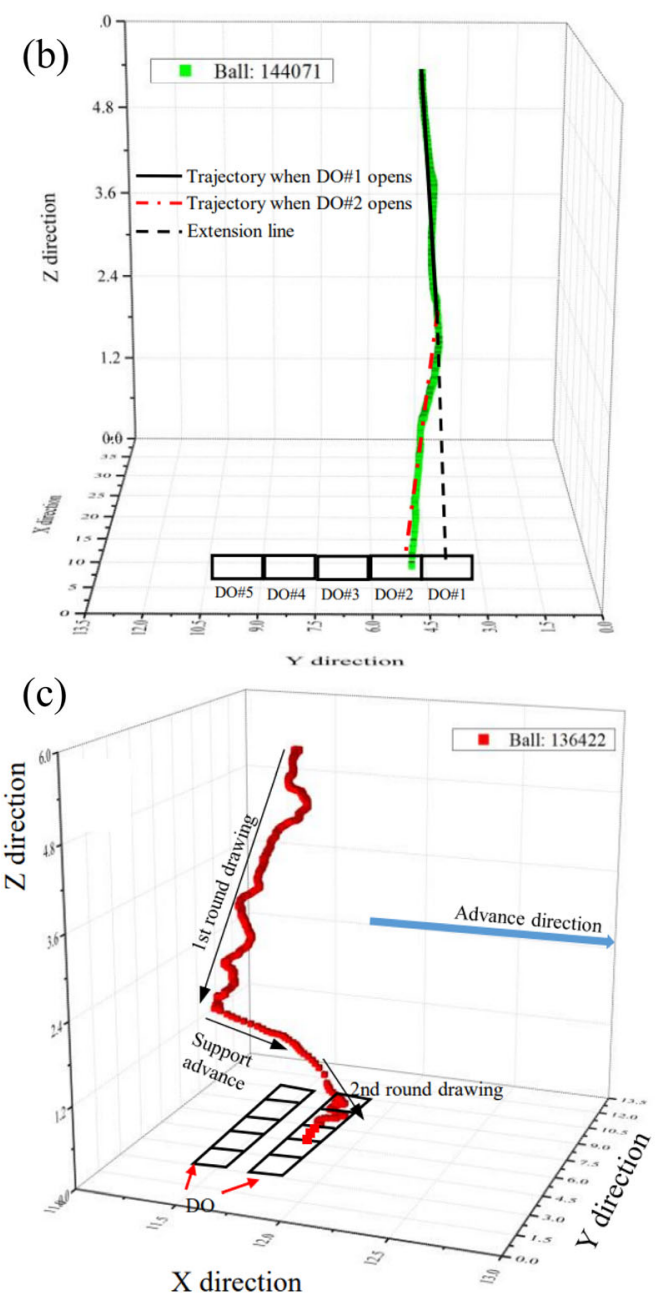
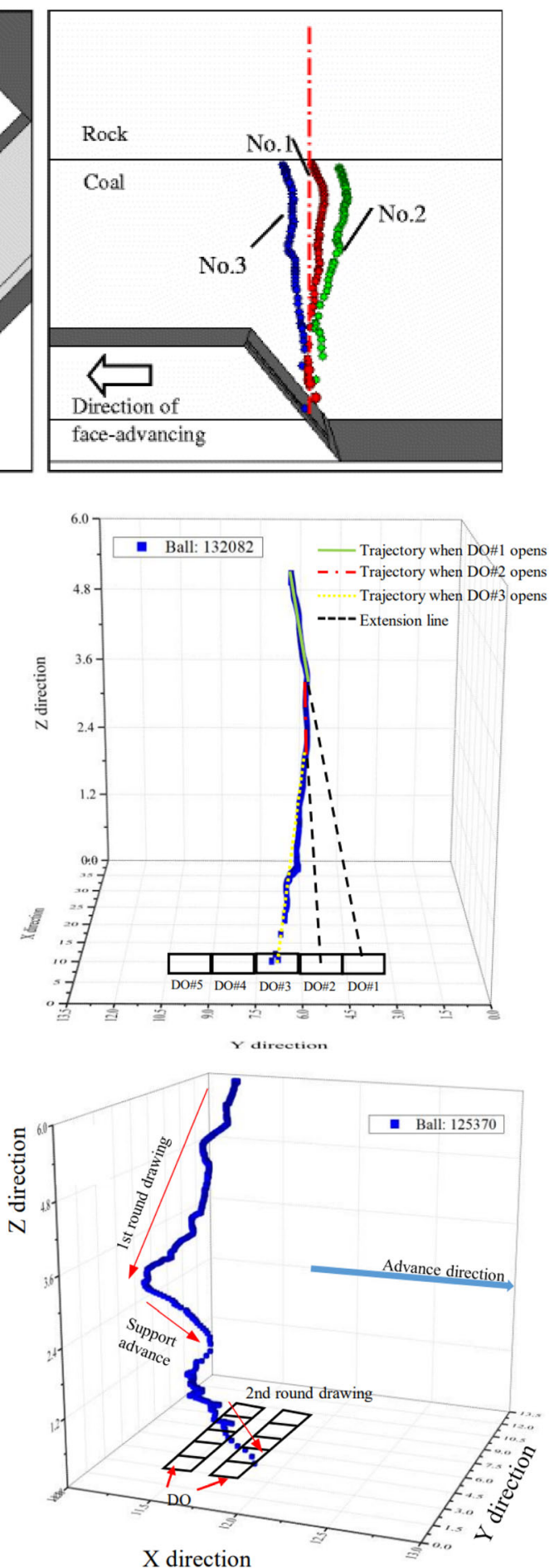

Fig. 18 Flow trajectories of top coal: a initial drawing, reproduced from Zhang et al. (2018a); b multi-drawing procedures; $\mathbf{c}$ two-round drawing with one DS advance; reproduced from Song and Konietzky (2019)

of the particles in the first drawing round are detailed. The three trajectories are approximately linear lines pointing to the DO, which is consistent with the assumptions of the B$\mathrm{R}$ model. The trajectory of top coal in multiple drawing rounds is shown in Fig. 18b (Song and Konietzky 2019), and signifies that particle trajectories subjected to the opening of at least two different DO. Figure $18 \mathrm{~b}$ shows that once two or more drawing procedures were used (Ball 144,071 had two drawing procedures and Ball 132,082 had three), the trajectories deviated from a straight line. The 
(a)
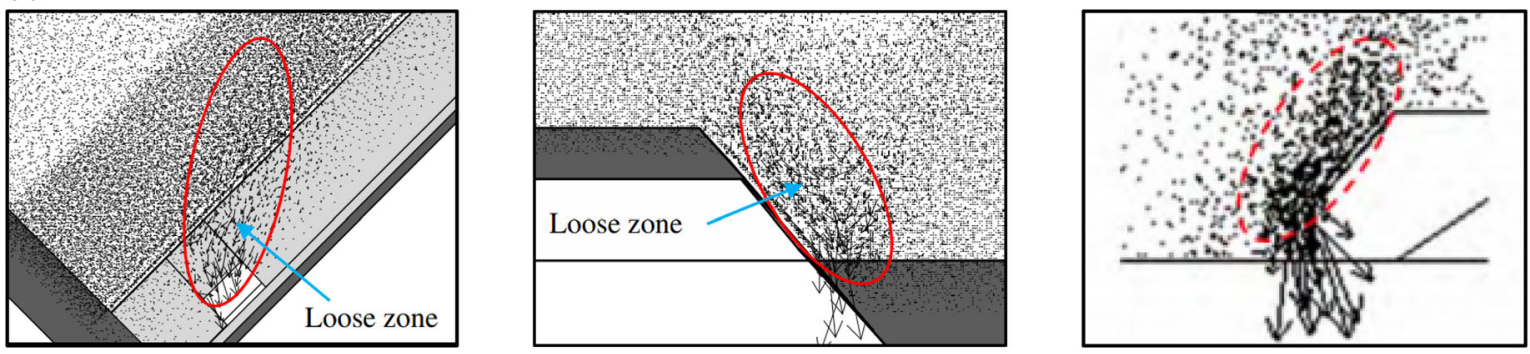

(b)

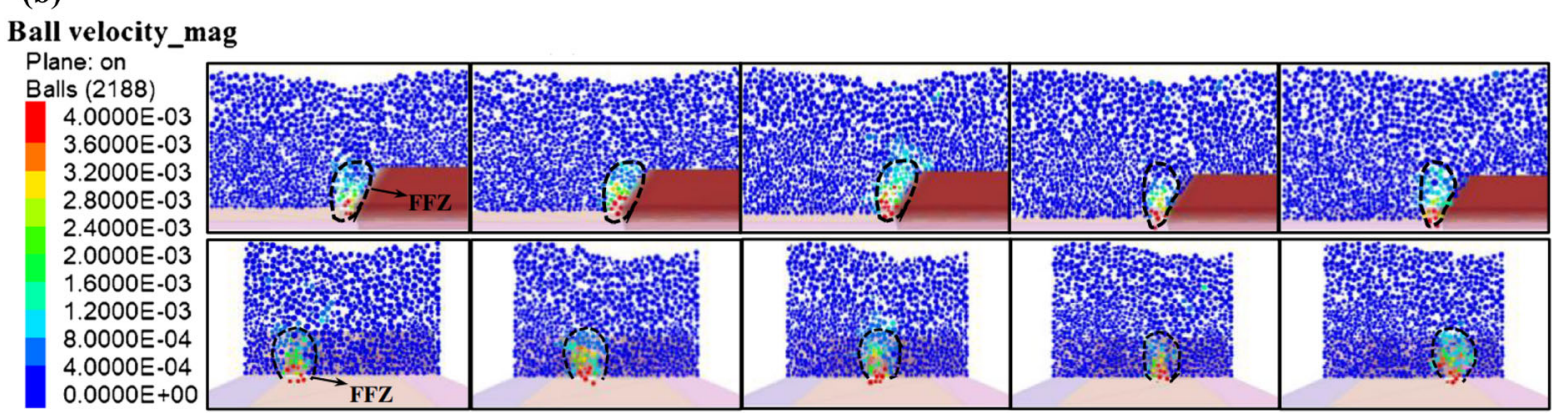

Fig. 19 Flow velocity field in numerical simulations: a indicated by the length of arrows, reproduced from Zhang et al. (2018a); b indicated by the colors, reproduced from Song and Konietzky (2019)

trajectory exposed to multiple drawing procedures can be divided into several sub-trajectories, where each points to the opened DO at the give time. For example, the particles in Fig. 18b respectively have two and three sub-trajectories, from which it is clear that each sub-trajectory points to the opened DO at the time. This shows that even a complete trajectory exposed to multiple drawing procedures is not a linear line, but the sub-trajectory still confirms the assumption (linear trajectory pointing to the DO) of the B$\mathrm{R}$ model. Figure 18c shows the trajectory of the particles by considering two drawing rounds and one support advance procedure (Song and Konietzky 2019). It is clear that the advance of the support causes the trajectory to develop in its direction. This indicates that the modeling of the support advance should be considered carefully in numerical simulations because it directly impacts the flow trajectory of top coal.

3.3.3.2 Flow velocity field The distribution of the velocity field of top coal can be used to characterize flow fluency during the drawing process. The arching always occurs in areas where the flow velocity is low, and can cause a jam. Figure 19 plots the distribution of the flow velocity of top coal during the drawing procedure (Wang and Zhang 2015; Zhang et al. 2018a; Song and Konietzky 2019). In Fig. 19a, the flow velocity is proportional to the length of the black arrows; a longer arrow indicates a higher flow velocity. The velocity drastically increases when the particles are closer to the DO. Furthermore, the velocity close to the rear canopy of the support is significantly higher than in any other area, and this can be attributed to the smooth contact between the top coal and the rear canopy of the support. This also leads to the deflection of the TCD presented in Sect. 3.3.1.1. Figure 19 plots the velocity field, which is indicated by different colors. A fluent flow zone (FFZ) is shown, indicating a higher flow velocity. The height of the FFZ is approximately equal to the height of the DS, which also verifies the effect of the rear canopy of the DS on the velocity field. The FFZ in Fig. 19b has a similar shape to the TCD reported in Sect. 3.3.1.1, which also exhibits a prominent deflection. 
Table 7 Practical applications of drawing mechanism to improve production

\begin{tabular}{|c|c|c|c|}
\hline Coal mine & Location & Geological characteristics & Guidance and improvements \\
\hline Dayuan & Shanxi & $\begin{array}{l}\text { 1. Steeply inclined coal seam with a maximum } \\
\text { inclination of } 62^{\circ} \\
\text { 2. The roof, coal seam and floor, were all soft }\end{array}$ & $\begin{array}{l}\text { 1. The in-situ TCRR was measured with the marker } \\
\text { method } \\
\text { 2. The top coal drawing sequence was optimized with } \\
\text { TCRR improved by } 8 \%\end{array}$ \\
\hline Luling & Anhui & $\begin{array}{l}\text { 1. Two coal seams (No. } 8 \text { and No. 9) were merged to use } \\
\text { LTCC } \\
\text { 2. The thickness of seam No. } 8 \text { was } 8.5 \mathrm{~m} \text { and that of } \\
\text { seam No. } 9 \text { was } 2.5 \mathrm{~m} \text {; the inclination of the coal seam } \\
\text { was } 12^{\circ}\end{array}$ & $\begin{array}{l}\text { 1. The in-situ TCRR was measured with the marker } \\
\text { method to determine the TCRR subjected to different } \\
\text { top coal layers } \\
\text { 2. The drawing interval and drawing sequence were } \\
\text { optimized with the TCRR improved by } 6.5 \%\end{array}$ \\
\hline Baojishan & Gansu & $\begin{array}{l}\text { 1. Thickness of the coal seam was } 11 \mathrm{~m} \text {, with the dip } \\
\text { angle varying in the range } 37-53^{\circ} \\
\text { 2. The lengths along the strike and dip directions were } \\
650 \mathrm{~m} \text { and } 112 \mathrm{~m} \text {, respectively }\end{array}$ & $\begin{array}{l}\text { 1. The in-situ TCRR was measured with the marker } \\
\text { method; the TCRR at different levels }(0-7 \mathrm{~m} \text { and } \\
7 \mathrm{~m}-11 \mathrm{~m}) \text { was measured } \\
\text { 2. The drawing interval and drawing sequence were } \\
\text { optimized with the TCRR improved by } 8 \%\end{array}$ \\
\hline Ruilong & Shanxi & $\begin{array}{l}\text { 1. Thickness of the coal seam varied in the range } \\
8-11 \mathrm{~m} \text {, with an average dip angle of } 20^{\circ} \\
\text { 2. The lengths along the strike and dip directions were } \\
513 \mathrm{~m} \text { and } 161 \mathrm{~m} \text {, respectively }\end{array}$ & $\begin{array}{l}\text { 1. The in-situ TCRR was measured with the marker } \\
\text { method, and the TCRR at different levels }(0-6 \mathrm{~m} \text { and } \\
6-9 \mathrm{~m}) \text { was measured } \\
\text { 2. Physical testing was conducted to investigate the } \\
\text { shapes of the TCB and TCD. The drawing interval and } \\
\text { drawing sequence were optimized with the TCRR } \\
\text { improved by } 6.7 \%\end{array}$ \\
\hline Wangzhuang & Shanxi & $\begin{array}{l}\text { 1. Average thickness of the coal seam was } 7.18 \mathrm{~m} \text { with } \\
\text { four layers of dirt bands } \\
\text { 2. The lengths along the strike and dip directions were } \\
955 \mathrm{~m} \text { and } 154.4 \mathrm{~m} \text {, respectively }\end{array}$ & $\begin{array}{l}\text { 1. The in-situ TCRR was measured with the marker } \\
\text { method } \\
\text { 2. A numerical simulation was conducted. The drawing } \\
\text { interval and drawing sequence were optimized with the } \\
\text { TCRR improved by } 7 \%\end{array}$ \\
\hline Tashan 8104 & Shanxi & $\begin{array}{l}\text { 1. The lengths along the strike and dip directions were } \\
570 \mathrm{~m} \text { and } 207 \mathrm{~m} \text {, respectively } \\
\text { 2. Thickness of the coal seam varied in the range } \\
12.90-16.44 \mathrm{~m} \text { with nine layers of dirt bands }\end{array}$ & $\begin{array}{l}\text { 1. The in-situ TCRR was measured with the marker } \\
\text { method } \\
\text { 2. Physical testing and numerical simulations were } \\
\text { conducted, and the effect of drawing interval on the } \\
\text { TCD and TCB was studied. The drawing interval and } \\
\text { drawing sequence were optimized with the TCRR } \\
\text { improved by } 17 \%\end{array}$ \\
\hline Tashan 8105 & Shanxi & $\begin{array}{l}\text { 1. The lengths along the strike and dip directions were } \\
2966 \mathrm{~m} \text { and } 207 \mathrm{~m} \text {, respectively, and the dip angle } \\
\text { varied in the range } 1-3^{\circ} \\
2 \text {. Thickness of the coal seam varied in the range } \\
9.42-19.44 \mathrm{~m}\end{array}$ & $\begin{array}{l}\text { 1. The in-situ TCRR was measured with the marker } \\
\text { method } \\
\text { 2. Physical testing and numerical simulations were } \\
\text { conducted to examine the effect of the drawing interval } \\
\text { on the TCD and TCB. The drawing interval and } \\
\text { drawing sequence were optimized with the TCRR } \\
\text { improved by } 13 \%\end{array}$ \\
\hline Xinjulong & Shandong & $\begin{array}{l}\text { 1. The lengths along the strike and dip directions were } \\
2570 \mathrm{~m} \text { and } 258 \mathrm{~m} \text {, respectively, and the dip angle } \\
\text { was in the range } 0-6^{\circ} \\
2 \text {. Thickness of the coal seam was in the range } \\
2.8-10.9 \mathrm{~m}\end{array}$ & $\begin{array}{l}\text { 1. The in-situ TCRR was measured with the marker } \\
\text { method } \\
\text { 2. Numerical simulations were conducted to examine the } \\
\text { effect of the drawing interval on the TCD and TCB. } \\
\text { The drawing interval and drawing sequence were } \\
\text { optimized with the TCRR improved by } 3 \%\end{array}$ \\
\hline Jiulishan & Henan & $\begin{array}{l}\text { 1. The lengths along the strike and dip directions were } \\
265 \mathrm{~m} \text { and } 172 \mathrm{~m} \text {, respectively } \\
\text { 2. Thickness of the coal seam thickness varies in the } \\
\text { range } 2-7 \mathrm{~m}\end{array}$ & $\begin{array}{l}\text { 1. The in-situ TCRR was measured with the marker } \\
\text { method } \\
\text { 2. Numerical simulation were conducted to examine the } \\
\text { effect of the drawing interval on the TCD and TCB. } \\
\text { The drawing interval and drawing sequence were } \\
\text { optimized with the TCRR improved by } 6 \%\end{array}$ \\
\hline
\end{tabular}


Table 7 continued

\begin{tabular}{|c|c|c|c|}
\hline Coal mine & Location & Geological characteristics & Guidance and improvements \\
\hline Wobei & Anhui & $\begin{array}{l}\text { 1. The lengths along the strike and dip directions were } \\
1371 \mathrm{~m} \text { and } 144 \mathrm{~m} \text {, respectively, and the dip angle } \\
\text { was } 13-27^{\circ} \\
\text { 2. Average thickness of the coal seam was close to } \\
6.36 \mathrm{~m}\end{array}$ & $\begin{array}{l}\text { 1. The in-situ TCRR was measured with the marker } \\
\text { method } \\
\text { 2. Numerical simulations were conducted to examine the } \\
\text { effect of the drawing interval on the TCD and TCB. } \\
\text { The drawing interval and drawing sequence were } \\
\text { optimized with the TCRR improved by } 5.7 \%\end{array}$ \\
\hline $\begin{array}{l}\text { Zhuxian } \\
\text { zhuang }\end{array}$ & Anhui & $\begin{array}{l}\text { 1. The lengths along the strike and dip directions were } \\
615 \mathrm{~m} \text { and } 171 \mathrm{~m} \text {, respectively, and the dip angle was } \\
5-15^{\circ} .2 \text {. Thickness of the coal seam thickness was } \\
3.8-30.6 \mathrm{~m} \text {, and the averaged thickness was close to } \\
16.5 \mathrm{~m}\end{array}$ & $\begin{array}{l}\text { The in-situ TCRR was measured with the marker } \\
\text { method. } 2 \text {. Numerical simulations were conducted to } \\
\text { examine the effect of the drawing interval on the TCD } \\
\text { and TCB. The drawing interval and drawing sequence } \\
\text { were optimized with the TCRR improved by } 4.8 \%\end{array}$ \\
\hline
\end{tabular}

\section{Practical applications of drawing mechanisms for LTCC mines}

The research system based on the framework documented in Fig. 2 can provide useful suggestions for optimization to improve production in LTCC mines. The results of physical testing and numerical simulations can be used to determine the appropriate drawing parameters, such as drawing sequences and drawing intervals, to obtain a higher TCRR. Table 7 presents the practical applications of drawing mechanisms to improve production in LTCC mines subjected to different geological characteristics based on more than 10 detailed cases investigated by our group over the last two decades. The guidance and improvements are listed in the right column, and verify that a fundamental understanding of the drawing mechanism of top coal is important for optimizing production in LTCC mines.

\section{Summary and perspectives}

\subsection{Summary}

This review presented and classified published research on the top coal drawing mechanism in the last two decades. It offers a comprehensive insight into the research frameworks, testing schemes, and modeling methods used to study the drawing mechanism in LTCC. LTCC is an advanced and promising mining technology for exacting ultra-thick coal seams, and research on the drawing mechanism of top coal has important implications for the application of LTCC. This section presents concluding remarks and some prospects for future work in the area.
The B-R model, kinematic model, and stochastic medium model can theoretically characterize the shape of the TCD. The modified B-R model is the most appropriate one to describe the shape of the TCD. The trajectory of flow of top coal is visualized using 3D numerical simulations, and is an approximately linear line point to the DO. The linear trajectory verifies the assumption of the $\mathrm{B}-\mathrm{R}$ model. The angle of the rear canopy, inclination of the coal seam, and sizes of top coal size and the DO all have direct impacts on the shape of the TCD. A larger dip angle and size of top coal will result in a larger and fatter TCD. Different drawing sequences and drawing intervals lead to distinct instances of the TCRR, alternate and multi-DO drawings are beneficial for enhancing it, drawing after three instances of DS advance yields the smallest TCRR, and an excessively large drawing interval leads to a greater loss of top coal. The velocity field of top coal is plotted, and top coal close to the rear canopy has a significantly higher flow velocity owing to the smooth contact between it and the rear canopy of the DS.

\subsection{Perspectives on future research}

Research on the drawing mechanism of top coal assumes that top coal is completely fractured at the outset. Such over-simplified assumptions neglect the progressive failure and fracture of top coal during extraction as well as continuous change in its size owing to secondary crushing induced by strata pressure. The precise measurement of onsite TCRR is still challenging for now; therefore, highly sensitive and portable measuring devices should be developed to precisely measure the TCRR on site. More theoretical models should be proposed to cater to more complex geological coal seams, such as seams with steep 
Table 8 Phenomena and mechanisms concerning the top coal drawing

\begin{tabular}{|c|c|c|c|}
\hline $\begin{array}{l}\text { Influencing } \\
\text { factor }\end{array}$ & Phenomena & Mechanism & $\begin{array}{l}\text { Direct or } \\
\text { indirect } \\
\text { factor }\end{array}$ \\
\hline $\begin{array}{l}\text { Angle of rear } \\
\text { canopy }\end{array}$ & $\begin{array}{l}\text { The angle of rear canopy can influence the } \\
\text { shape of TCD }\end{array}$ & $\begin{array}{l}\text { The angle of rear canopy determines the flowing boundary of the } \\
\text { top coal along the side of rear canopy, which means that a } \\
\text { steeper angle of rear canopy can result in a more limited zone } \\
\text { when the top coal flows out. The angle of rear canopy is } \\
\text { directly related to the maximum allowable moving angle } \theta_{G} \text { in } \\
\text { Bergmark-Roos model. Consequently, the variation of } \theta_{G} \\
\text { induced by the angle of rear canopy is the root reason to } \\
\text { influence the shape of TCD }\end{array}$ & $\begin{array}{l}\text { Direct } \\
\text { factor }\end{array}$ \\
\hline $\begin{array}{l}\text { Inclination } \\
\text { angle of the } \\
\text { coal seam }\end{array}$ & $\begin{array}{l}\text { The inclination of the coal seam will } \\
\text { influence the shape of TCD }\end{array}$ & $\begin{array}{l}\text { The inclination of the coal seam determines the top boundary of } \\
\text { the flowing field for fractured top coal. This is proportional to } \\
\text { the height of TCD. Normally, with the increase of the seam } \\
\text { inclination, the maximum flowing height will increase as a } \\
\text { result. This will result in more drawn-out top coal blocks from } \\
\text { the top part of the working face. However, the inclination will } \\
\text { also cause the asymmetrical TCD. The TCD will incline to the } \\
\text { upper side of the working face. In summary, the inclination of } \\
\text { coal seam equivalently increases the height of TCD }\end{array}$ & $\begin{array}{l}\text { Direct } \\
\text { factor }\end{array}$ \\
\hline Size of top coal & $\begin{array}{l}\text { The size of top coal will influence the shape } \\
\text { of TCD }\end{array}$ & $\begin{array}{l}\text { The repose angles of top coal particles with different sizes are } \\
\text { distinct. In a certain range, the repose angle decreases with } \\
\text { increasing top coal sizes. According to the mathematic } \\
\text { relationship between the repose angle and } \theta_{G} \text { in Bergmark- } \\
\text { Roos model, it can be obtained that } \theta_{G} \text { gradually increases } \\
\text { with the increase of top coal size, which results in a fatter and } \\
\text { larger TCD. Therefore, the variation of top coal size changes } \\
\text { the physical and mechanical properties of the loose top coal } \\
\text { blocks, mainly refers to the change of repose angle and } \\
\text { interlocking }\end{array}$ & $\begin{array}{l}\text { Direct } \\
\text { factor }\end{array}$ \\
\hline Size of DO & $\begin{array}{l}\text { The size of DO will influence the shape of } \\
\text { TCD and TCRR }\end{array}$ & $\begin{array}{l}\text { As the boundary condition of top coal drawing, the change of } \\
\text { DO's size directly affects the TCD shape, and its essence is to } \\
\text { change the range and volume of drawn-out top coal. In } \\
\text { general, with rising the length of DO, } \theta_{G} \text { and the size of top } \\
\text { coal gradually increase, which makes the volume of TCD } \\
\text { gradually rises. However, considering the influence of other } \\
\text { factors, TCRR increases gradually with increasing size of DO } \\
\text { in extra-thick coal seams. However, for the thinner coal } \\
\text { seams, TCRR tends to decrease with increasing the size of } \\
\text { DO, which is due to the top coal loss between supports } \\
\text { resulted from the increase of } \theta_{G}\end{array}$ & $\begin{array}{l}\text { Direct } \\
\text { factor }\end{array}$ \\
\hline $\begin{array}{l}\text { Sequence of } \\
\text { top coal } \\
\text { drawing }\end{array}$ & $\begin{array}{l}\text { The optimal drawing sequence should be } \\
\text { determined according to the specific } \\
\text { geological conditions }\end{array}$ & $\begin{array}{l}\text { The sequence of top coal drawing will influence the location as } \\
\text { well as the size of DO, the variations have obvious impacts on } \\
\text { the flowing fields of the fractured top coal }\end{array}$ & $\begin{array}{l}\text { Indirect } \\
\text { factor }\end{array}$ \\
\hline $\begin{array}{l}\text { Top coal } \\
\text { drawing } \\
\text { interval }\end{array}$ & $\begin{array}{l}\text { The optimal drawing interval should be } \\
\text { determined according to the specific } \\
\text { geological conditions }\end{array}$ & $\begin{array}{l}\text { The drawing interval will affect the shape of TCB, and then } \\
\text { change the interaction between TCB and TCD. Specifically, } \\
\text { for normal coal seams, increasing drawing interval will reduce } \\
\text { the tangent range between TCB and TCD, and increase the } \\
\text { volume of top coal loss in the goaf, which is not beneficial to } \\
\text { improve TCRR. However, in the extra-thick coal seams, the } \\
\text { increase of drawing interval is beneficial to the extraction of } \\
\text { upper top coal and can improve TCRR in LTCC panels }\end{array}$ & $\begin{array}{l}\text { Indirect } \\
\text { factor }\end{array}$ \\
\hline
\end{tabular}


inclination and variations in thickness. Both physical testing and numerical simulations should be performed while considering the pressure of the strata during the drawing process. The development of modeling that couples the finite element method and the discrete element method would be an interesting approach in this vein.

Authors contribution The author contributions include: JW proposed the research framework and the structure of the manuscript, and reviewed the manuscript. ZS and WW conducted the literature review and wrote the manuscript. SY and JZ reviewed and revised the manuscript.

Funding This work is funded by the National Natural Science Foundation of China (Grant No. 51934008, 51674264 to Jiachen Wang; Grant No. 51974320 to Shengli Yang), Fundamental Research Funds for the Central Universities (Grant No. 06500182 to Zhengyang Song), Funds from State Key Laboratory of Coal Resources in Western China (SKLCRKF20-07 to Zhengyang Song).

Data availability The data can be available from the authors upon request.

Code availability The code can be available from the authors upon request.

\section{Declaration}

Conflict of interest The authors declare that they have no conflict of interest.

Open Access This article is licensed under a Creative Commons Attribution 4.0 International License, which permits use, sharing, adaptation, distribution and reproduction in any medium or format, as long as you give appropriate credit to the original author(s) and the source, provide a link to the Creative Commons licence, and indicate if changes were made. The images or other third party material in this article are included in the article's Creative Commons licence, unless indicated otherwise in a credit line to the material. If material is not included in the article's Creative Commons licence and your intended use is not permitted by statutory regulation or exceeds the permitted use, you will need to obtain permission directly from the copyright holder. To view a copy of this licence, visit http://creativecommons. org/licenses/by/4.0/.

\section{References}

Aghababaei S, Jalalifar H, Saeedi G (2019) Prediction of face advance rate and determination of the operation efficiency in retreat longwall mining panel using rock engineering system. Int J Coal Sci Technol 6(3):419-429

Alehossein H, Poulsen BA (2010) Stress analysis of longwall top coal caving. Int J Rock Mech Min Sci 47(1):30-41

Bai Q, Tu S, Wang C (2014) Numerical simulation on top-coal arching mechanism. Jounrnal Min Saf Eng 31(2):208-213

Bai Q, Tu S, Wang F (2018) Characterizing the top coal cavability with hard stone band(s): insights from laboratory physical modeling. Rock Mech Rock Eng 52(5):1505-1521
Castro R, Gómez R, Hekmat A (2016) Experimental quantification of hang-up for block caving applications. Int J Rock Mech Min Sci 85:1-9

Chen Q, Zhao F, Chen Q et al (2017) Orthogonal simulation experiment for flow characteristics of ore in ore drawing and influencing factors in a single funnel under a flexible isolation layer. JOM 69(12):2485-2491

Hao X, Du W, Zhao Y et al (2020) Dynamic tensile behaviour and crack propagation of coal under coupled static-dynamic loading. Int J Min Sci Technol 30(5):659-668

Huang B, Liu C, Wu F, Wang Z (2006) Experimental research on drawing top-coal with loose medium model under dead-unconsolidated sandstone roof. J China Univ Min Technol 35(3):351-355

Huang B, Liu C, Cheng Q (2007) Relation between top-coal drawing ratio and refuse content for fully mechanized top coal carving. J China Coal Soc 32(8):789-793

Huang B, Liu C, Niu H, Wang J (2008) Research on coal-gangue flow field character resulted from great cutting height fully mechanized top coal caving. J Min Saf Eng 25(4):415-419

Huang B, Wang Y, Cao S (2015) Cavability control by hydraulic fracturing for top coal caving in hard thick coal seams. Int $\mathbf{J}$ Rock Mech Min Sci 74:45-57

Huang B, Cheng Q, Zhao X, Kang C (2018) Hydraulic fracturing of hard top coal and roof for controlling gas during the initial mining stages in longwall top coal caving: a case study. J Geophys Eng 15(6):2492-2506

Huo Y, Song X, Zhu D (2020) Numerical investigation of top-coal migration in the first coal-drawing process by an FDM-DEM coupling method. Energ 13(20):5493

Jangara H, Ozturk CA (2021) Longwall top coal caving design for thick coal seam in very poor strength surrounding strata. Int $\mathrm{J}$ Coal Sci Technol. https://doi.org/10.1007/s40789-020-00397-y

Jin A, Sun H, Ma G et al (2016) A study on the draw laws of caved ore and rock using the discrete element method. Comput Geotech 80:59-70

Jin A, Sun H, Wu S, Gao Y (2017) Confirmation of the upside-down drop shape theory in gravity flow and development of a new empirical equation to calculate the shape. Int J Rock Mech Min Sci 92:91-98

Klishin SV, Klishin VI, Opruk GY (2013) Modeling coal discharge in mechanized steep and thick coal mining. J Min Sci 49(6):932-940

Klishin1 SV, Klishin VI, Opruk GY (2018) Discrete element modeling of gravity flow of broken rocks in the technology of longwall top coal caving. IOP Conf Ser Earth Environ Science, vol 206, IOP Publishing, p 012007

Kuchta M (2002) A revised form of the Bergmark-Roos equation for describing the gravity flow of broken rock. Miner Resour Eng 11:349-360

Lai X, Shan P, Cao J et al (2014) Hybrid assessment of pre-blasting weakening to horizontal section top coal caving (HSTCC) in steep and thick seams. Int J Min Sci Technol 24(1):31-37

Le TD, Mitra R, Oh J, Hebblewhite B (2017) A review of cavability evaluation in longwall top coal caving. Int J Min Sci Technol 27(6):907-915

Le TD, Oh J, Hebblewhite B et al (2018) A discontinuum modelling approach for investigation of longwall top coal caving mechanisms. Int J Rock Mech Min Sci 106:84-95

Le TD, Zhang C, Oh J et al (2019) A new cavability assessment for longwall top coal caving from discontinuum numerical analysis. Int J Rock Mech Min Sci 115:11-20

Li S, Fan C, Luo M et al (2017) Structure and deformation measurements of shallow overburden during top coal caving longwall mining. Int J Min Sci Technol 27(6):1081-1085 
Litwiniszyn J (1956) Application of the equation of stochastic processes to mechanics of loose bodies. Arch Mech Stosow 8(4):393-411

Litwiniszyn J (1963) The model of a random walk of particles adopted to researches on problems of mechanics of granular. Bull Acad Pol Sci Ser Sci Technol 9:61

Liu B, Zhang J (1995) Stochastic method for ground subsidence due to near surface excavation. Chin J Rock Mech Eng 14(4):289-295

Liu C, Huang B, Wu F (2009) Technical parameters of drawing and coal-gangue field movements of a fully mechanized large mining height top coal caving working face. Min Sci Technol 19(5):549-555

Melo F, Vivanco F, Fuentes C, Apablaza V (2007) On drawbody shapes: from Bergmark-Roos to kinematic models. Int J Rock Mech Min Sci 44(1):77-86

Melo F, Vivanco F, Fuentes C, Apablaza V (2008) Kinematic model for quasi static granular displacements in block caving: dilatancy effects on drawbody shapes. Int $\mathrm{J}$ Rock Mech Min Sci 45(2):248-259

Melo F, Vivanco F, Fuentes C (2009) Calculated isolated extracted and movement zones compared to scaled models for block caving. Int J Rock Mech Min Sci 46(4):731-737

Miao S, Lai X, Cui F (2011) Top coal flows in an excavation disturbed zone of high section top coal caving of an extremely steep and thick seam. Min Sci Technol 21(1):99-105

Nedderman RM, Tüzün U (1979) A kinematic model for the flow of granular materials. Powder Technol 22(2):243-253

Qiao D, Sun Y, Ren F (2003) Study on movement probability density equation of ore-drawing stochastic theory. J China Coal Soc 28(4):361-365

Rustan A (2000) Gravity flow of broken rock-what is known and unknown. In: Proceedings of MassMin 2000, Brisbane

Sánchez V, Castro RL, Palma S (2019) Gravity flow characterization of fine granular material for Block Caving. Int J Rock Mech Min Sci 114:24-32

Sharrock GB (2008) The Isolated Extraction Zone in Block Caving A Review. In: Potvin Y, Carter J, Dyskin A, Jeffrey R (eds) Proceedings of the First Southern Hemisphere International Rock Mechanics Symposium. Australian Centre for Geomechanics, pp 255-271

Si G, Jamnikar S, Lazar J et al (2015) Monitoring and modelling of gas dynamics in multi-level longwall top coal caving of ultrathick coal seams, part I: Borehole measurements and a conceptual model for gas emission zones. Int $\mathrm{J}$ Coal Geol 144-145:98-110

Simsir F, Ozfirat MK (2008) Determination of the most effective longwall equipment combination in longwall top coal caving (LTCC) method by simulation modelling. Int J Rock Mech Min Sci 45(6): 1015-1023

Song Z, Konietzky H (2019) A particle-based numerical investigation on longwall top coal caving mining. Arab J Geosci 12(18):556

Song Z, Wei W, Zhang J (2018) Numerical investigation of effect of particle shape on isolated extracted zone (IEZ) in block caving. Arab J Geosci 11(12):310

Song Z, Konietzky H, Herbst M (2020) Drawing mechanism of fractured top coal in longwall top coal caving. Int J Rock Mech Min Sci 130(1):104329

Tao G, Yang S, Liu Z (2010) Research of ore drawing method of broken ore and rock based on Bergmark-Roos equation. J China Coal Soc 35(5):750-753

Vakili A, Hebblewhite BK (2010) A new cavability assessment criterion for Longwall Top Coal Caving. Int J Rock Mech Min Sci 47:1317-1329

Wang J (2008) Study on coal rock caving laws and technological parameters of top coal caving of fully-mechanized mining with great cutting height for thick coal seam. Ph.D. Thesis, China University of Mining and Technology

Wang J (2014) Development and prospect on fully mechanized mining in Chinese coal mines. Int $\mathrm{J}$ Coal Sci Technol 1(3):253-260

Wang J (2018) Engineering practice and theoretical progress of topcoal caving mining technology in China. J China Coal Soc 43(1):43-51

Wang J, Song Z (2015) Characteristic and control method of initial interface between coal and rock under fully mechanized loose top coal caving. J China Coal Eng 47(4):1-4

Wang J, Zhang J (2015) BBR study of top-coal drawing law in longwall top-coal caving mining. J China Coal Soc 40(3):487-493

Wang J, Wei L, Zhang J, Li Z, Wang JC, Wei LK, Zhang JW et al (2013) 3D numerical simulation on the top-coal movement law under caving mining technique. J China Coal Soc 38(11):1905-1911

Wang J, Yang S, Li Y et al (2014) Caving mechanisms of loose topcoal in longwall top-coal caving mining method. Int $\mathrm{J}$ Rock Mech Min Sci 71:160-170. https://doi.org/10.1016/j.ijrmms. 2014.04.024

Wang J, Yang S, Zhang J, Song Z (2015a) 3-D movement law of topcoal in near horizontal coal seam with multi-gangue under caving mining technique. J China Coal Soc 40(5):979-987

Wang J, Yu B, Kang H et al (2015b) Key technologies and equipment for a fully mechanized top-coal caving operation with a large mining height at ultra-thick coal seams. Int J Coal Sci Technol 2(2):97-161

Wang J, Zhang J, Li Y (2015) Ground control in China's coal mine: Progress and prospects. In: 49th US Rock Mechanics / Geomechanics Symposium, p 240

Wang J, Zhang J, Song Z, Li Z (2015d) Three-dimensional experimental study of loose top-coal drawing law for longwall top-coal caving mining technology. J Rock Mech Geotech Eng 7(3):318-326

Wang J, Chen Y, Zhang J (2016a) Optimization study on drawing technique of longwall top-coal caving in extra-thick coal seam based on BBR system. Coal Eng 48(2):1-4

Wang J, Song Z, Zhang J, Chen Y (2016b) Theoretical model of drawing body in LTCC mining. J China Coal Soc 41(2):352-358

Wang J, Zhang J, Chen Y (2016c) Research on technology of improving top-coal recovery in longwall top-coal caving mining based on BBR system. J Min Sci Technol 1(1):38-48

Wang J, Zhang J, Li Z (2016d) A new research system for caving mechanism analysis and its application to sublevel top-coal caving mining. Int J Rock Mech Min Sci 88:273-285

Wang J, Zhang J, Wang Z (2018a) Basic theories and applications in top-coal caving mining. Science Press, Beijing

Wang Z, Wang J, Yang S (2018b) An ultrasonic-based method for longwall top-coal cavability assessment. Int J Rock Mech Min Sci 112:209-225

Wang J, Wei W, Zhang J (2019a) Effect of the size distribution of granular top coal on the drawing mechanism in LTCC. Granul Matter 21(3):70

Wang J, Wei W, Zhang J (2019b) Theoretical description of drawing body shape in an inclined seam with longwall top coal caving mining. Int J Coal Sci Technol 7(1):182-195

Wang J, Wei W, Zhang J et al (2020a) Numerical investigation on the caving mechanism with different standard deviations of top coal block size in LTCC. Int J Min Sci Technol 30(5):583-591

Wang J, Wei W, Zhang J, Mishra B (2020b) Laboratory and field validation of a LTCC recovery prediction model using relative size of the top coal blocks. Bull Eng Geol Environ 80(2):1389-1401 
Wang J, Yang L, Li F, Wang C (2020c) Force chains in top coal caving mining. Int J Rock Mech Min Sci. https://doi.org/10. 1016/j.ijrmms.2020.104218

Wei W, Song Z, Zhang J (2018) Theoretical equation of initial topcoal boundary in longwall top-coal caving mining. Int J Min Miner Eng 9(3):157-176

Xie Y-S, Zhao Y-S (2009a) Numerical simulation of the top coal caving process using the discrete element method. Int J Rock Mech Min Sci 46:983-991

Xie Y, Zhao Y (2009b) Technique of top coal caving with vibration. Procedia Earth Planet Sci 1:219-226

Yang S, Zhang J, Chen Y, Song Z (2016) Effect of upward angle on the drawing mechanism in longwall top-coal caving mining. Int $J$ Rock Mech Min Sci 85:92-101

Yasitli NE, Unver B (2005) 3D numerical modeling of longwall mining with top-coal caving. Int $\mathrm{J}$ Rock Mech Min Sci 42:219-235

Yu B, Zhu D, Chen Z (2017) Top-coal drawing law of LTCC mining based on stochastic medium theory. J China Coal Soc 42(6):1366-1371

Zhang N, Liu C (2016) Arch structure effect of the coal gangue flow of the fully mechanized caving in special thick coal seam and its impact on the loss of top coal. Int J Min Sci Technol 26(4):593-599

Zhang ZX, Wimmer M (2018) A case study of dividing a single blast into two parts in sublevel caving. Int $\mathrm{J}$ Rock Mech Min Sci 104:84-93

Zhang J, Song Z, Geng H (2014) Numerical simulation on top-coal movement law under caving mining technique in steeply dipping seam influenced by caving sequence. In: Transit Development in
Rock Mechanics-Recognition, Thinking and Innovation - Proceedings of the 3rd ISRM Young Scholars Symposium on Rock Mechanics. pp 313-318

Zhang J, Pan W, Li Z, Song Z (2015a) Development and application of $3 \mathrm{D}$ simulation test device for loose top-coal drawing under caving mining technique. Chinese J Rock Mech Eng 34(S2):3871-3879

Zhang N, Liu C, Pei M (2015b) Effects of caving-mining ratio on the coal and waste rocks gangue flows and the amount of cyclically caved coal in fully mechanized mining of super-thick coal seams. Int J Min Sci Technol 25(1):145-150

Zhang N, Liu C, Yang P (2016) Flow of top coal and roof rock and loss of top coal in fully mechanized top coal caving mining of extra thick coal seams. Arab J Geosci 9(6):465

Zhang J, Wang J, Wei W et al (2018a) Experimental and numerical investigation on coal drawing from thick steep seam with longwall top coal caving mining. Arab J Geosci 11(5):96

Zhang J, Wang J, Wei W (2018b) Effect of face dip angle on the drawing mechanism in longwall top-coal caving mining. J China Unversity Min Technol 47(4):805-814

Zhang N, Liu C, Wu X, Ren T (2018c) Dynamic Random Arching in the Flow Field of Top-Coal Caving Mining. Energ 11(5):1106

Zhang Q, Yue J, Liu C et al (2019) Study of automated top-coal caving in extra-thick coal seams using the continuum-discontinuum element method. Int J Rock Mech Min Sci 122(1):104033

Zhu D, Chen Z, Du W et al (2018) Caving mechanisms of loose topcoal in longwall top-coal caving mining based on stochastic medium theory. Arab J Geosci 11(20):621 